\title{
A decade of SN 1993J: discovery of radio wavelength effects in the expansion rate
}

\author{
J. M. Marcaide ${ }^{1}$, I. Martí-Vidal ${ }^{1,2}$, A. Alberdi ${ }^{3}$, M. A. Pérez-Torres ${ }^{3}$, E. Ros ${ }^{2,1}$, P. J. Diamond ${ }^{4}$, J. C. Guirado ${ }^{1}$, \\ ${\text { L. } \text { Lara }^{\dagger 5} \text {, I. I. Shapiro }}^{6}$, C. J. Stockdale ${ }^{7}$, K. W. Weiler ${ }^{8}$, F. Mantovani ${ }^{9}$, R. A. Preston ${ }^{10}$, R. T. Schilizzi ${ }^{11}$, \\ R. A. Sramek ${ }^{12}$, C. Trigilio ${ }^{13}$, S. D. Van Dyk ${ }^{14}$, and A. R. Whitney ${ }^{15}$ \\ 1 Departamento de Astronomía, Universidad de Valencia, Valencia, Spain \\ e-mail: J.M.Marcaide@uv. es \\ 2 Max-Planck-Institut für Radioastronomie, Bonn, Germany \\ 3 Instituto de Astrofísica de Andalucía, CSIC, Granada, Spain \\ 4 Jodrell Bank Observatory, University of Manchester, Manchester, England \\ Deceased; Universidad de Granada, Granada, Spain \\ ${ }^{6}$ Harvard-Smithsonian Center for Astrophysics, Cambridge, MA, USA \\ 7 Marquette University, Milwaukee, WI, USA \\ 8 Naval Research Laboratory, Washington D.C., USA \\ 9 Istituto di Radioastronomia, INAF, Bologna, Italy \\ 10 Jet Propulsion Laboratory, NASA, Pasadena, CA, USA \\ 11 International SKA Project Office, Dwingeloo, The Netherlands \\ 12 National Radio Astronomy Observatory, Socorro, NM, USA \\ 13 Istituto di Radioastronomia, INAF, Noto, Italy \\ 14 Spitzer Science Center, Caltech, Pasadena, CA, USA \\ 15 Haystack Observatory, MIT, Westford, MA, USA
}

Received 23 March 2009 / Accepted 27 April 2009

ABSTRACT

\begin{abstract}
We studied the growth of the shell-like radio structure of supernova SN 1993J in M 81 from September 1993 to October 2003 with very-long-baseline interferometry (VLBI) observations at the wavelengths of $3.6,6$, and $18 \mathrm{~cm}$. We developed a method to accurately determine the outer radius $(R)$ of any circularly symmetric compact radio structure such as SN 1993J.

The source structure of SN 1993J remains circularly symmetric (with deviations from circularity under 2\%) over almost 4000 days. We characterize the decelerated expansion of SN 1993J until approximately day 1500 after explosion with an expansion parameter $m=0.845 \pm 0.005\left(R \propto t^{m}\right)$. However, from that day onwards the expansion differs when observed at 6 and $18 \mathrm{~cm}$. Indeed, at $18 \mathrm{~cm}$, the expansion can be well characterized by the same $m$ as before day 1500, while at $6 \mathrm{~cm}$ the expansion appears more decelerated, and is characterized by another expansion parameter, $m_{6}=0.788 \pm 0.015$. Therefore, since about day 1500 onwards, the radio source size has been progressively smaller at $6 \mathrm{~cm}$ than at $18 \mathrm{~cm}$. These findings differ significantly from those of other authors in the details of the expansion. In our interpretation, the supernova expands with a single expansion parameter, $m=0.845 \pm 0.005$, and the $6 \mathrm{~cm}$ results beyond day 1500 are caused by physical effects, perhaps also coupled to instrumental limitations. Two physical effects may be involved: (a) a changing opacity of the ejecta to the $6 \mathrm{~cm}$ radiation; and (b) a radial decrease of the magnetic field in the emitting region.

We also found that at $6 \mathrm{~cm}$ about $80 \%$ of the radio emission from the backside of the shell behind the ejecta is absorbed (our average estimate, since we cannot determine any possible evolution of the opacity), and the width of the radio shell is (31 \pm 2$) \%$ of the outer radius. The shell width at $18 \mathrm{~cm}$ depends on the degree of assumed absorption. For $80 \%$ absorption, the width is (33.5 \pm 1.7$) \%$, and for $100 \%$ absorption, it is $(37.8 \pm 1.3) \%$.

A comparison of our VLBI results with optical spectral line velocities shows that the deceleration is more pronounced in the radio than in the optical. This difference might be due to a progressive penetration of ejecta instabilities into the shocked circumstellar medium, as also suggested by other authors.
\end{abstract}

Key words. galaxies: clusters: individual: M 81 - radio continuum: stars - supernovae: general - supernovae: individual: SN 1993J techniques: interferometric

\section{Introduction}

Supernova SN 1993J was visually discovered in the nearby galaxy M 81 on 28 March 1993 by García (Ripero \& García 1993). It reached $m_{v}=10.8$ and became the brightest supernova in the northern hemisphere since SN 1954A (see Matheson et al. $2000 \mathrm{~b}$ and references therein). The relatively small distance to M 81 (3.6 Mpc, Freedman et al. 1994) and the high northern declination of M 81 soon made SN 1993J one of the best observed supernova ever, and particularly so at very high angular resolution. Although initially classified as Type II (Filippenko et al. 1993), it did not behave like other Type II -plateau or linear supernovae. Its light curve showed two peaks separated by about 2 weeks.

The unusual initial behavior of the light curve led many modelers to conclude that SN 1993J was the result of a 
core-collapse explosion of a progenitor that had lost a significant fraction of its hydrogen envelope, leaving less than one solar mass of hydrogen in the core. Mass-loss from a massive star through winds was proposed by Höflich et al. (1993), an explosion of an asymptotic giant branch star of smaller main sequence mass with a helium-rich envelope was proposed by Hashimoto et al. (1993), and stripping of hydrogen by a companion in a binary system was proposed by many other modelers. Models of the light curve and spectra (Nomoto et al. 1993; Filippenko et al. 1993; Schmidt et al. 1993; Swartz et al. 1993; Wheeler et al. 1993; Podsiadlowski et al. 1993; Ray et al. 1993; Taniguchi et al. 1993; Shigeyama et al. 1994; Utrobin 1994; Bartunov et al. 1994; and Woosley et al. 1994) suggested ejecta masses in the range $2-6 M_{\odot}$, with only $0.1-0.9 M_{\odot}$ in a thin outer hydrogen envelope with an initial radius of several hundred solar radii. The first maximum in the optical light curve was interpreted as being caused by shock heating of the thin envelope and the second maximum by the radioactive decay of ${ }^{56} \mathrm{Co}$.

Later studies continued to suggest that a low-mass envelope of hydrogen on a helium core was the most likely scenario for the progenitor (Young et al. 1995; Patat et al. 1995; Utrobin 1996; Houck \& Fransson 1996). The low-mass outer layer of hydrogen would give the initial appearance of a Type II, but the spectrum would slowly change to one more similar to that of a Type Ib, as had already been considered by Woosley et al. (1987) and Filippenko (1988) for SN 1987K. Following Woosley et al. (1987), SN 1993J is of Type IIb.

The binary system scenario also received support from presupernova photometry of the region, which indicated the presence of more than one star (Aldering et al. 1994). According to Filippenko et al. (1993), the progenitor was probably a giant of type K0 I in a binary system. When, much later, the companion to the progenitor was discovered (Maund et al. 2004), the binary system scenario received final backing.

Trammell et al. (1993) and Tran et al. (1997) found optical continuum polarization from SN 1993J at the level of $1 \%$ and argued that the polarization implied an overall asymmetry, although the source of the asymmetry was not identified. The presence of SN $1993 \mathrm{~J}$ in a binary system provided a plausible source of the asymmetry.

Models of early spectra reproduced their overall shape (Baron et al. 1993), but had difficulties fitting line strengths (Baron et al. 1994; Jeffery et al. 1994; Clocchiatti et al. 1995). Wang \& Hu (1994), Spyromilio (1994), and Matheson et al. (2000a) argued in favor of clumpy ejecta.

An early UV spectrum taken with the HST by Jeffery et al. (1994) showed a smooth spectrum similar to SN 1979C and SN 1980K, both of which were also radio sources. Branch et al. (2000) suggested that the illumination from circumstellar interaction might reduce the relative strengths of line features and produce featureless UV spectra. Indeed, the presence of circumstellar interaction could be clearly seen in late nebular-phase spectra (Filippenko et al. 1994; Li et al. 1994; Barbon et al. 1995; Finn et al. 1995) with $\mathrm{H}_{\alpha}$ lines beginning to dominate the spectrum. Both Patat et al. (1995) and Houck \& Fransson (1996) concluded that the late-time optical spectra could only be powered by a circumstellar interaction, since radioactive decay seemed to be insufficient.

Further support for circumstellar interaction came from the early detection of X-rays (Zimmerman et al. 1994; Kohmura et al. 1994). Those X-rays could come from either the shocked wind material or from the reverse-shocked supernova ejecta (Suzuki \& Nomoto 1995; Fransson et al. 1996), according to the standard circumstellar interaction model (SCIM) for supernovae.
The SCIM considers supernova ejecta with steep density profiles $\left(\rho_{\mathrm{ej}} \propto r^{-n}\right)$ shocked by a reverse shock that moves inward (in a Lagrangian sense) from the contact surface, and a circumstellar medium (CSM) with density profile $\rho_{\mathrm{CSM}} \propto r^{-s}$ shocked by a forward shock that moves outward from the contact surface ( $s=2$ corresponds to a steady wind). For $n>5$, self-similar solutions are possible (Chevalier 1982b); the radii of the discontinuity surface, forward shock, and reverse shock are then related, and all evolve in time with the power law $R \propto t^{m}$, where $t$ is the time after explosion and $m$ is the deceleration parameter, which is determined by $n$ and $s$ in terms of the expression $m=(n-3) /(n-s)$. In this model, radio emission would arise from the shocked region between the supernova ejecta and the CSM resulting from the wind of the supernova's progenitor star (Chevalier 1982a).

Radio emission at $2 \mathrm{~cm}$ from SN 1993J was detected within two weeks after the explosion by Pooley \& Green (1993) and soon light curves were available at 1.3, 2, 3.6, 6, and $20 \mathrm{~cm}$ (Van Dyk et al. 1994). The high level of radio emission from this supernova and its high northern declination paved the way for a superb sequence of very-long-baseline interferometry (VLBI) observations, which started very early on (Marcaide et al. 1994; Bartel et al. 1994) and have continued for over a decade. From VLBI observations, Marcaide et al. (1995b) found a spherically symmetric shell of width about 0.3 times the outer radius, and Marcaide et al. (1995a) showed the first movie of the self-similar growth of the shell over one year. With VLBI data from three years of observations, Marcaide et al. (1997) reported deceleration in the expansion of the shell and estimated a value of the deceleration parameter $m=0.86 \pm 0.02$. Combining this estimate with the determination of the opacity due to free-free absorption in the CSM by Van Dyk et al. (1994), we derived a value of $s=1.66_{-0.25}^{+0.12}$ (Marcaide et al. 1997) in agreement with the value $s=1.7$ given by Fransson et al. (1996) to explain the X-ray emission. However, such a determination of the freefree opacity, and hence of the value of $s$, has been questioned by Fransson \& Björnsson (1998) who instead argued in favor of $s=2$ and emphasized the importance of synchrotron selfabsorption. Pérez-Torres et al. (2001) also emphasized the importance of synchrotron self-absorption in the interpretation of the radio light curves.

The determination of the deceleration parameter also allows for a direct comparison of ejecta density profiles determined from modelling the emission spectrum. Using NLTE algorithms, Baron et al. (1995) derived a value of $n=50$ shortly after the explosion, decreasing to $n=10$ at late epochs. The later $n$ corresponds (for $s=2$ ) to $m=0.875$, compatible with the determination by Marcaide et al. (1997). Additionally, such a value of $m$ is compatible (for the assumed distance of $3.6 \mathrm{Mpc}$ to M 81) with the expansion speeds of $14000 \mathrm{~km} \mathrm{~s}^{-1}$ up to 1000 days after explosion (Garnavich \& Ann 1994) and of $10000 \mathrm{~km} \mathrm{~s}^{-1}$ for days 1000-1400 after explosion (Fransson et al. 2005). The radio spectrum at long wavelengths has been studied by Pérez-Torres et al. (2002), and Chandra et al. (2004). Fransson \& Björnsson (1998) proposed a model in which the size of the radio emitting region would be discernibly wavelength dependent. Those longwavelength results and the Fransson \& Björnsson model will be considered in Sect. 7.1.1.

The expansion of the radio shell has taken place with remarkable spherical symmetry (Marcaide et al. 1995a, 1997, this paper; Bietenholtz et al. 2001, 2003, 2005; Alberdi \& Marcaide 2005). This result, while complying nicely with the simplest SCIM, is in sharp contrast with the claims of asymmetry (Trammell et al. 1993; Tran et al. 1997) based on the 
detection of optical polarization from the supernova ejecta, which require a ratio of about 0.6 for the radii of an elliptical emission model. It is hard to imagine that the ejecta could have such an asymmetry and the outer shock front, as delineated by the outer surface of the radio emission, such a remarkable symmetry. These characteristics would appear to be inconsistent with the SCIM. Perhaps, as pointed out by Matheson et al. (2000b), there is no such inconsistency between the early optical polarimetric observations and the VLBI observations since the two types of observations may probe different regions of the supernova shell.

Great effort has been invested in determining the width of the expanding radio shell and the value of $m$ as a function of time after explosion by two groups working on independently acquired VLBI data. Each group has used different data acquisition and analysis strategies. Bartel et al. (2002) confirmed the deceleration reported earlier by Marcaide et al. (1997), but claimed that the values of $m$ differ for different expansion periods. Those results were in agreement with previous results from numerical simulations made by Mioduszewski et al. (2001) using a rather specific explosion model. Preliminary observational evidence to the contrary was provided by Marcaide (2005) and definitive evidence is provided in this paper. After the initial estimate by Marcaide et al. (1995b) of a shell width of $0.3 \pm 0.1$ times the size of the outer radius of the source, Bartel et al. (2000) reported shell widths as narrow as $0.205 \pm 0.015$. However, after Bietenholz et al. (2003), and Marcaide et al. (2005) provided evidence of absorption in the central part of the shell emission, Bietenholz et al. (2005) revised their estimates of the shell width to $0.25 \pm 0.03$, consistent with the value reported by Marcaide et al. (1995b), and closer to, but still inconsistent with, the more accurate estimate reported in this paper.

The study of SN 1993J has been very important for at least two reasons: a) for the first time a clear transition from Type II to Type Ib was observed, thus linking Type Ib (and for that matter Type Ic) to massive core collapse supernovae, such as Type II, rather than the thermonuclear explosion supernovae, such as Type Ia; and b) for the first time a long sequence of images following a supernova were obtained to provide detailed information on the expansion rate. The results from such monitoring have lent support to the SCIM initially proposed by Chevalier (1982a,b). However, Bartel et al. (2002) claim to have detected departures from a self-similar expansion with regimes of changing expansion rates over different periods. In this paper, we provide evidence contrary to such claims based on our own data and on the use of new analysis tools, and support the validity of the SCIM model in which additional fine observational effects have to be taken into account.

The remainder of this paper is organized as follows: we first describe our observations. Then we describe a novel approach to improved imaging and measuring of source size and shell width, and compare the results obtained with different methods. We give tentative physical and observational reasons for the rather surprising result that the apparent expansion rate at two wavelengths is slightly, but significantly, different. Finally, assuming the distance to SN 1993J as that obtained by Freedman et al. (1994) for M 81, we compare radio and optical results.

\section{Observations, correlation, and data reduction}

Table 1 summarizes all our VLBI observations of SN 1993J at 3.6, 6, and $18 \mathrm{~cm}$ from 1993 September 26 through 2003 October 17 . Our early results at 3.6 and $6 \mathrm{~cm}$ were published by Marcaide et al. (1995a,b, 1997). In this paper, we reanalyze those published data together with the new data using new analysis methods that will be described below.

The antennas that participated in all or some of our observations are: the VLBA (10 identical antennas of $25 \mathrm{~m}$ diameter each spread over the US from the Virgin Islands to Hawaii), the phased-VLA (equivalent area to a paraboloid of $130 \mathrm{~m} \mathrm{di-}$ ameter, New Mexico, USA), the Green Bank Telescope (100 m, WV, USA), Goldstone (70 m, CA, USA), Robledo (70 m, Spain), and the European VLBI Network including Effelsberg $(100 \mathrm{~m}$, Germany), Medicina (32 m, Italy), Noto (32 m, Italy), Jodrell Bank (76m, UK), Onsala (20 and $25 \mathrm{~m}$, Onsala, Sweden), Westerbork (equivalent area to a paraboloid of $93 \mathrm{~m}$ diameter, The Netherlands). The Goldstone and Robledo antennas could only take part in the 3.6 and $18 \mathrm{~cm}$ observations. Westerbork only took part in the 6 and $18 \mathrm{~cm}$ observations. The effective array consisted typically of about 15 antennas. The recording was each time set to the maximum available rate at that time (256 Mbps), 2-bit sampling, single polarization mode (RCP at $3.6 \mathrm{~cm}$ and LCP at 6 and $18 \mathrm{~cm}$ ). The synthesized bandwidth was $64 \mathrm{MHz}$ (except at the VLA, where it was limited to $50 \mathrm{MHz}$ ). The data were correlated either at the Max Planck Institut für Radioastronomie, Bonn, Germany, when the MkIV recording system was used (see Marcaide et al. 1997), or at the National Radio Astronomy Observatory, Socorro, NM, USA, when the VLBA recording was used (after 1997 February 2). We provide in Table 1 a summary of the VLBI observations including the rms noise of each of the reconstructed maps of SN 1993J.

A typical 12h observation cycled between SN 1993J and the core of M 81, and observed occasionally 0917+624 and 0954+658. Additionally, we observed a number of sources such as 3C 286 and 3C 48 at appropriate times during each observing session for flux-density calibration purposes. Once the correlated data were available, we initially calibrated the data with the radiometry information obtained at each antenna participating in the array. For all data reduction purposes apart from mapping, we used the NRAO AIPS package, and for mapping we used DIFMAP (Shepherd et al. 1995).

We usually started the data reduction by analyzing the $0917+624$ and $0954+658$ data. We used these two sources for instrumental calibration (we ran program FRING on data integrated over the duration of a scan to determine the residual delays which aligned the 16 channels of the IF for $0917+624$ and 0954+658.) After previously reducing the residual fringe rates to a weighted mean of zero, we applied those residual delays to data from the whole observing session and, in particular, searched for new residual phase-delay and residual delay-rate solutions for the core of M 81, integrating the data over the duration of each scan and assuming a centered point model for M 81. Finally, we mapped the core of M 81 in DIFMAP using, whenever necessary, phase and amplitude self-calibration. With the map of the core of M 81 at hand, we used it as input to programs FRING and CALIB of AIPS to improve the fringe search solution by removing the contribution of the phases due to the structure of the core of M 81 from the data stream, and to improve the amplitude calibration, respectively. The new fringe solution for the complete data set (and for SN 1993J, in particular) is thus referred to the reference point chosen in the core of M 81. We finally time averaged the SN 1993J data over 2 minutes and frequency averaged over the synthesized band.

\section{Imaging of SN1993J}

Once the SN 1993J data had been calibrated in AIPS as described in the previous section, the mapping of SN 1993J was 
Table 1. Summary of VLBI observations.

\begin{tabular}{crcccc|c|c|c}
\hline \hline $\begin{array}{c}\text { Date } \\
(\mathrm{dd} / \mathrm{mm} / \mathrm{yy})\end{array}$ & $\begin{array}{c}\text { Age }^{a} \\
(\text { days })\end{array}$ & $\begin{array}{c}\text { Map Flux }^{b} \\
(\mathrm{mJy})\end{array}$ & $\begin{array}{c}\text { Map Peak }^{c} \\
\left(\mathrm{mJy} \mathrm{beam}^{-1}\right)\end{array}$ & $\begin{array}{c}\text { Noise rms }^{d} \\
\left(\mathrm{mJy} \mathrm{beam}^{-1}\right)\end{array}$ & $\begin{array}{c}\text { Radius }^{e} \\
(\mathrm{mas})\end{array}$ & \multicolumn{3}{|c}{$\lambda^{1}(\mathrm{~cm})$} \\
\hline $26 / 09 / 93$ & 182 & 78.50 & 8.91 & 0.037 & $0.488 \pm 0.004$ & $\mathrm{X}$ & & \\
$22 / 11 / 93$ & 239 & 57.30 & 7.32 & 0.089 & $0.628 \pm 0.014$ & $\mathrm{X}$ & & \\
$20 / 02 / 94$ & 329 & 51.00 & 6.52 & 0.037 & $0.818 \pm 0.015$ & $\mathrm{X}$ & & \\
$29 / 05 / 94$ & 427 & 41.50 & 5.24 & 0.094 & $1.02 \pm 0.02$ & $\mathrm{X}$ & & \\
$20 / 09 / 94$ & 541 & 53.40 & 7.01 & 0.097 & $1.15 \pm 0.03$ & & $\mathrm{X}$ & \\
$23 / 02 / 95$ & 697 & 44.30 & 5.53 & 0.090 & $1.48 \pm 0.04$ & & $\mathrm{X}$ & \\
$11 / 05 / 95$ & 774 & 41.80 & 5.63 & 0.083 & $1.66 \pm 0.04$ & & $\mathrm{X}$ & \\
$01 / 10 / 95$ & 917 & 32.20 & 4.36 & 0.170 & $1.92 \pm 0.04$ & & $\mathrm{X}$ & \\
$28 / 03 / 96$ & 1096 & 31.30 & 3.47 & 0.082 & $2.21 \pm 0.03$ & & $\mathrm{X}$ & \\
$17 / 06 / 96$ & 1177 & 26.50 & 2.94 & 0.110 & $2.31 \pm 0.04$ & & $\mathrm{X}$ & \\
$22 / 10 / 96$ & 1304 & 26.10 & 3.12 & 0.074 & $2.61 \pm 0.02$ & & $\mathrm{X}$ & \\
$25 / 02 / 97$ & 1430 & 24.44 & 2.46 & 0.085 & $2.81 \pm 0.05$ & & $\mathrm{X}$ & \\
$21 / 09 / 97$ & 1638 & 21.75 & 2.52 & 0.061 & $3.09 \pm 0.03$ & & $\mathrm{X}$ & \\
$18 / 02 / 98$ & 1788 & 21.28 & 2.28 & 0.061 & $3.37 \pm 0.03$ & & $\mathrm{X}$ & \\
$30 / 05 / 98$ & 1889 & 22.24 & 2.94 & 0.059 & $3.48 \pm 0.04$ & & $\mathrm{X}$ & \\
$23 / 11 / 98$ & 2066 & 20.62 & 2.30 & 0.045 & $3.74 \pm 0.04$ & & $\mathrm{X}$ & \\
$30 / 11 / 98$ & 2073 & 39.84 & 4.67 & 0.050 & $3.84 \pm 0.06$ & & & $\mathrm{X}$ \\
$10 / 06 / 99$ & 2265 & 18.05 & 1.89 & 0.053 & $4.06 \pm 0.04$ & & $\mathrm{X}$ & \\
$22 / 09 / 99$ & 2369 & 17.02 & 2.03 & 0.043 & $4.20 \pm 0.04$ & & $\mathrm{X}$ & \\
$06 / 06 / 00$ & 2627 & 15.87 & 2.19 & 0.066 & $4.48 \pm 0.06$ & & $\mathrm{X}$ & \\
$20 / 11 / 00$ & 2794 & 27.72 & 3.34 & 0.040 & $4.98 \pm 0.07$ & & & $\mathrm{X}$ \\
$24 / 11 / 00$ & 2798 & 14.24 & 1.64 & 0.047 & $4.71 \pm 0.10$ & & $\mathrm{X}$ & \\
$14 / 02 / 01$ & 2880 & 13.10 & 1.37 & 0.057 & $4.84 \pm 0.07$ & & $\mathrm{X}$ & \\
$18 / 11 / 01$ & 3157 & 10.58 & 1.28 & 0.048 & $5.21 \pm 0.10$ & & $\mathrm{X}$ & \\
$07 / 11 / 02$ & 3511 & 10.36 & 1.11 & 0.047 & $5.67 \pm 0.10$ & & $\mathrm{X}$ & \\
$17 / 11 / 02$ & 3521 & 16.12 & 1.80 & 0.050 & $6.06 \pm 0.05$ & & & $\mathrm{X}$ \\
$29 / 10 / 03$ & 3867 & 09.47 & 1.25 & 0.046 & $6.15 \pm 0.11$ & & $\mathrm{X}$ & \\
\hline & & & & &
\end{tabular}

${ }^{a}$ Age is in days after explosion.

${ }^{b}$ Map Flux corresponds to total map flux density.

${ }^{c}$ Maximum flux density per unit beam in the maps.

${ }^{d}$ Root-mean-square of the corresponding residual maps.

${ }^{e}$ SN 1993J radius (and its standard deviation) as estimated using the Common-Point Method described in the text (see Sect. 4.2 and Appendix A for details).

completed in DIFMAP. Especial care was taken during the imaging process to avoid introducing any bias that might affect the final estimate of the SN 1993J radius. Data that had been analyzed earlier, and the results already published (Marcaide et al. 1995a,b, 1997), were also reanalyzed with this new approach.

Since the structure of SN 1993J is very circularly symmetric, the visibilities, when referred to the center of the map, are such that the imaginary part cancels out. All the information is then contained in the real part of the visibility. We used this property to determine the position of the center of the map with data from day 1889 after explosion (i.e., 1998 May 30; see Table 1). We chose this map because of its very high quality. Its center, determined with respect to the core of M 81 for this epoch, was used for every epoch. For each epoch, we found that the imaginary parts of the visibilities were compatible with zero. Since this condition was satisfied for every epoch, we concluded that the structure remained circularly symmetric and that the center of the structure remained stationary with respect to the core of M 81.

To be consistent with the use of a dynamic beam, which was introduced by Marcaide et al. (1997) (see also next section), to avoid a bias in the measurement of the supernova expansion, a similar use of a dirty dynamic beam had to be made during the imaging process. This use was achieved by tapering the data with a Gaussian taper, whose width evolved inversely with the source size. In particular, taking advantage of the azimuthal symmetry of the source, we used at all epochs a normalized Gaussian taper such that its half value falls at the middle of the third lobe of the visibility function, as shown schematically in Fig. 1.

Another innovation of our imaging process is the use of a non-point source initial model in the mapping process. Customarily, a point source model is taken as the initial model for imaging of radio sources. However, such a choice is not the best one for the mapping of SN 1993J. Instead, we have taken advantage of SN 1993J being rather circularly symmetric to design a procedure that is objective and very useful in the fine calibration of the visibilities in the imaging process. We now describe this procedure in detail.

Due to the circular symmetry of the source and its relatively sharp edge, the visibilities display very clear lobes and the details of the source structure are most evident in the second and higher order lobes. Indeed, the Fourier transform of a perfectly circularly symmetric source will be real. In such a case, the phases will alternate between 0 and $\pi$ radians in the lobes, being zero 


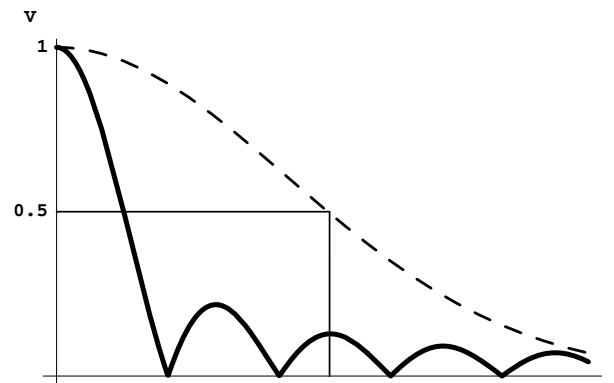

Fig. 1. Schematic representation of the taper used in our mapping and model-fitting procedures. The dark continuous line represents the amplitude of the visibilities, while the dashed one represents the taper. The point indicated on the dashed line corresponds to the value of the uvradius such that the taper value is equal to 0.5 . We have chosen this value to correspond to the middle of the third lobe of the visibility amplitudes. As the supernova expands, the lobes will shrink in the $u v$-plane as will the taper function, thus increasing the width of the dirty beam in the same proportion as the radius.

for the first lobe. We can use this circumstance to our benefit in the case of SN 1993J since, as noted above, it is rather circularly symmetric, with irregularities being of a small scale perceptible only in the higher-order lobes. Thus, if we can guess the location of the transition between the first and second lobes, then, using only the data from those two lobes, we can adopt for self-calibration a perfectly symmetric model, which has its first-to-second-lobe transition at roughly the same point as for the data. We can thus use the data that correspond to the first two lobes for the initial phase self-calibration, ignoring the data corresponding to higher resolution. This self-calibration with the program SELFCAL will force the data to have 0 phase for the first lobe and $\pi$ for the second. Given that the solutions obtained will be antenna dependent, a new self-calibration step, now using all the data, will clearly define the locations of the remaining phase changes (from 0 to $\pi$, or viceversa) for higher resolution data.

We note that the quality of the initial guess in the location of the transition between the first and second lobes, and the use of different models for the initial self-calibration, are not crucial to the procedure. The former is true because near the null the phases are ill defined in all cases, while the latter is true because the procedure has to do with phases, and is therefore insensitive to the amplitudes of the model. We verified the correctness of the previous assertions. In practice, we used a simple (uniformly bright) disc model for the initial self-calibration of the data in the first two lobes.

After self-calibrating the phase data, we proceeded in the usual way of mapping, using iterative low gain CLEANing and phase self-calibration a number of times. As a final step, we applied amplitude and phase calibration for every $30 \mathrm{~min}$ of data.

In Fig. 2, we show the contour maps for observations made in or near October of every year. These maps are representative of all the maps we have reconstructed ${ }^{2}$.

\section{Measurement of the radius of SN 1993J}

\subsection{Introduction}

The circular shape of the images of SN 1993J facilitates the task of defining a radius. Even so, since an accurate value of the

\footnotetext{
${ }^{2}$ Color maps can be found in the following web page: http://www . uv. es/radioastronomia/SN1993J-10yr-AA09.jpg.
}

radius of SN 1993J at each epoch is crucial to study the details of the expansion, especial care has to be taken in the estimate of the radius. Before we describe our present approach, we note that Marcaide et al. (1997) and Bartel et al. (2000) took different approaches to estimating the radius in their attempts to determine the details of the expansion. Marcaide et al. (1997) used the average of the radial distances from the map center to the $50 \%$ contour level of the maps for a number of directions. To avoid a bias in the radii estimates, these authors had convolved the source models with beams proportional to supernova sizes to obtain the maps. Instead, Bartel et al. (2000) estimated the supernova outer radius (as well as the inner radius) by fitting a shell model in Fourier space. For this purpose, they assumed a specific, spherical, optically-thin source model.

In this work, we tried to overcome the drawbacks of each of the previous measuring schemes. The principal drawback of the procedure of Bartel et al. (2000) was that the estimates were model-dependent. This drawback became apparent when it was found later that the emission from the central part of the source is greatly suppressed (Bartel et al. 2002; Marcaide et al. 2005). We refined the procedure of Marcaide et al. (1997) by developing new tools that allow for accurate measurements on the sky plane, while keeping the measuring scheme model-independent.

Before describing our method in detail, we illustrate it with two simple one-dimensional cases: (a) We consider a uniform source whose emission intensity is nonzero for all $r \leq R$ and zero for $r>R$ (that is, the source is the equivalent of a disk in 2 dimensions). We convolve this step-function source with Gaussian beams of different widths, $\sigma$, such that in every case $\sigma$ is much smaller than $R$. After convolution, all resulting functions will cross at $R$ at half the height of the step function. Thus, the crossing point of the resultant functions exactly determines the source radius $R$. (b) Secondly, we consider a narrow "boxcar" source, that is, a uniform source whose emission is nonzero only over a narrow region just short of its outer edge, $r=R$ (that is, the equivalent to a thin shell in 2 dimensions). If we convolve this model of the source with the Gaussian beams $\sigma$ described above, we find that the center of the resultant function will almost coincide with $R$, but the position of the outer half-power point will be larger than $R$.

For a one-dimensional model in-between the above extreme models (e.g., a "boxcar" of width comparable to the values of $\sigma$ (that is, the equivalent of a thick shell in 2 dimensions)), using the outer half-power point of the resultant function to determine the radius $R$ would give a result $r>R$. The ratio $r / R$ would remain constant provided the model maintained its functional shape while changing $R$ (self-similar change) and provided $\sigma$ changed fractionally the same amount as $R$.

This latter, intermediate, one-dimensional model illustrates the idea of the Marcaide et al. (1997) method: use of dynamical beams to reconstruct the SN 1993J images before measuring their sizes at the $50 \%$ contour level. While each of the size measurements might be slightly biased, the expansion measured will not be biased provided that the shape of the source emission does not change with time. Since the central absorption (found later) in SN 1993J appears to have been strong at all times, the expansion results given by Marcaide et al. (1997) are likely to be nearly unbiased. The expansion measured with Bartel et al.'s method (fitting a model to the visibilities) is likely to be biased since use of an incorrect model (optically thin, without central absorption) will bias each measurement of the radius, and likely in a time-dependent manner as the amount of the visibility sidelobes involved in the fit changes as the source grows in size. The Common Point Method described in the next section has, in this 


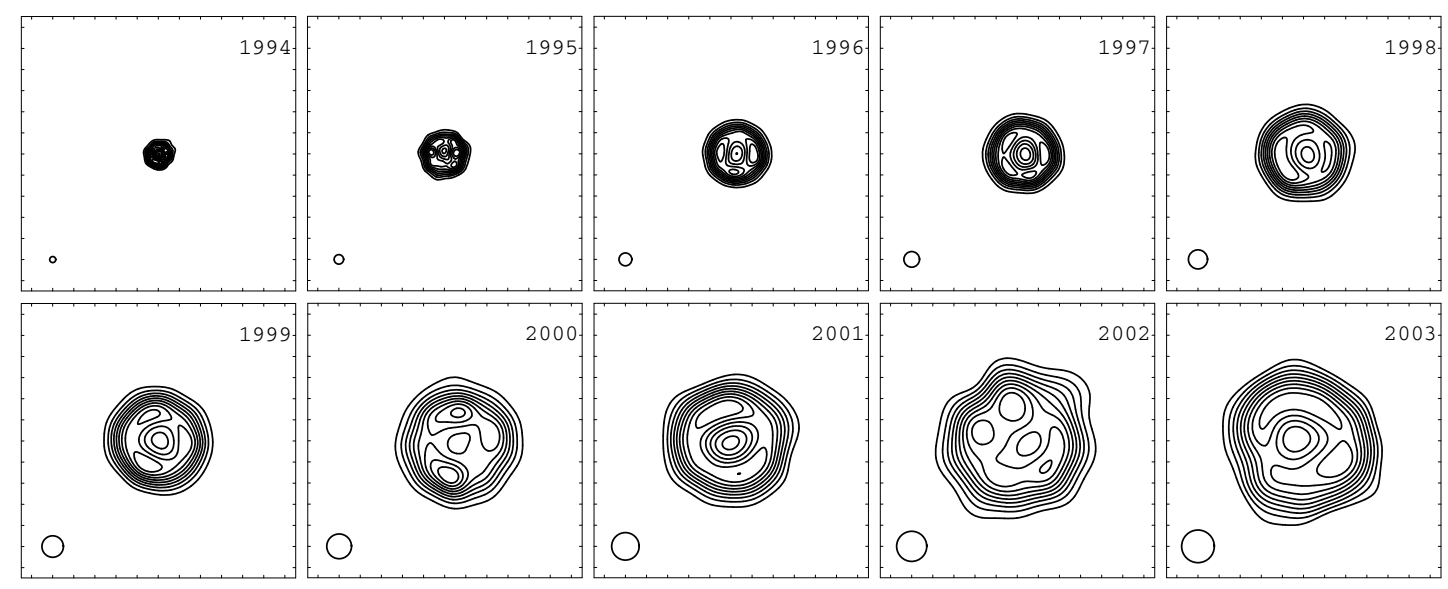

Fig. 2. Maps of SN 1993J at $6 \mathrm{~cm}$ corresponding to epochs in or near October every year from 1994 through 2003. The FWHM of the circular beam used to reconstruct each map is shown in the lower left corner. Contours correspond to $(10,20,30,40,50,60,70,80,90) \%$ of peak emission (see Table 1). Tick marks are in milliarcseconds (mas). One mas in each map corresponds to approximately 3500 AU.

respect, the same advantages as the method used by Marcaide et al. (1997) but is more accurate.

\subsection{The Common Point Method}

Given a map of SN 1993J, if we azimuthally average its brightness distribution we obtain a profile similar to that shown in Fig. 3 (solid line). For maps corresponding to the same model but reconstructed with beams of different sizes, different profiles will be obtained. However, if those profiles are superimposed to each other we find that they cross approximately at two points, as shown in Fig. 3. We call "outer common point" (OCP) the outer approximate common point for all profiles. (We take the name of the method from this characteristic.) We use the radial distance of the OCP, $X_{\mathrm{OCP}}$, as an estimate of the source radius. We call "inner common point" (ICP) the inner approximate common point for all profiles. The position of the ICP, $X_{\mathrm{ICP}}$, is related to the inner shell border.

In practice, we reconstruct a map using a beam of size (full width at half maximum, FWHM) equal to half the source radius, $X_{\mathrm{OCP}}$, and iterate until the estimate of the source radius changes fractionally in an iteration by less than 0.01 ; three iterations are usually sufficient to determine the source size. The final estimate of $X_{\mathrm{OCP}}$ is our CPM estimate of the source size. The uncertainty in this estimate is assumed to be related to the lack of circularity of the source (see Appendix A).

The whole method relies strongly on the properties of the outer common point. Because of this reliance, we included in Appendix A a mathematical description of the method and details about how the method works in practice.

\subsection{Considerations on the use of the Common Point Method}

Simulations can be used to investigate the biases in the size determination with the CPM. The number of simulations can be reduced considerably by taking into account what is already known about the source emission structure. Previous VLBI observations provided strong support to a shell-like structure of the emission, although there is not as yet agreement on the size of the shell width (Marcaide et al. 1995a; Bartel et al. 2000). There is also evidence that part of the emission from an otherwise optically thin shell is suppressed (Bartel et al. 2002; Marcaide et al. 2005); it appears that the emission from the part of the shell

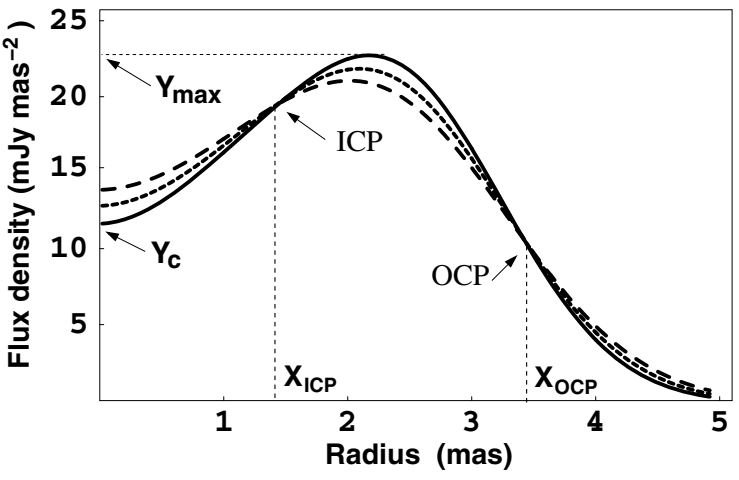

Fig. 3. (Solid line) Profile obtained azimuthally averaging the map obtained at $6 \mathrm{~cm}$ from observations made on day 1889 after explosion (see Fig. A.1a in Appendix A) for a convolving beam size of 1.74 mas. Tick marks are in mas ( $X$ axis) and in $\mathrm{mJy} \mathrm{mas}^{-2}$ ( $Y$ axis). $Y_{\max }$ is the value of the profile at maximum and $Y_{\mathrm{c}}$ is the value of the profile at the source center. The dotted and dashed lines are profiles obtained in the same way but using convolving beam sizes of 1.92 and 2.1 mas, respectively. The three profiles cross at two points; the position of the outer one $\left(X_{\mathrm{OCP}}\right)$ is taken to be the source size in the CPM; the difference between it and the inner one $\left(X_{\mathrm{OCP}}-X_{\mathrm{ICP}}\right)$ is taken to be related to the shell width of the source.

behind the ejecta is very much absorbed. Hence, we considered two classes of models to test the accuracy and bias of the CPM. One class of models consists of an optically thin shell, and the other consists of an optically thin shell with the emission from behind the ejecta suppressed by absorption. Furthermore, for each class we considered in our simulations 3 different shell widths: $0.25,0.30$, and 0.35 times the outer radius, $R$. The relevant information in each case can be extracted by considering 4 significant points in profiles such as those in Fig. 3: $Y_{\max }$, the value of profile at maximum; $Y_{\mathrm{c}}$, the value of the profile at the source center; $X_{\mathrm{ICP}}$, the radial position of the inner common point; and $X_{\mathrm{OCP}}$, the radial position of the outer common point.

$X_{\mathrm{OCP}}$ is our estimate of the model radius. Is this estimate unbiased? No. It is biased by a factor that depends on the source structure. Table 2 shows the ratio of $X_{\mathrm{OCP}}$ and the true radius for each model in our simulations. Two more ratios carry significant information about the model structure: the ratio $\rho_{1}=\frac{Y_{c}}{Y_{\max }}$ gives an indication of the absorption. The larger the absorption the smaller $Y_{\mathrm{c}}$ will be. Given that $Y_{\max }$ is less sensitive than $Y_{\mathrm{c}}$ to the 


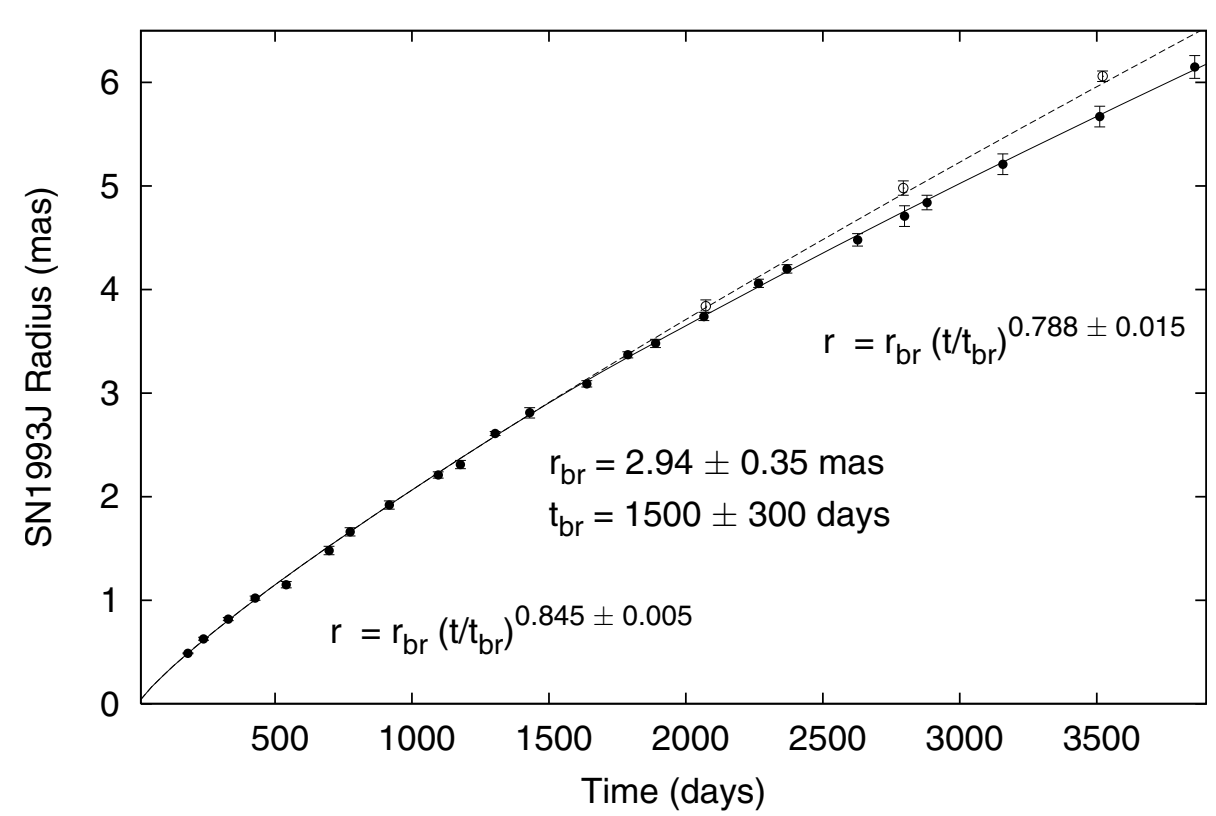

Fig. 4. Expansion of SN 1993J over a decade, measured from the estimated date of explosion. Filled circles represent 3.6 and $6 \mathrm{~cm}$ data and empty circles $18 \mathrm{~cm}$ data. The continuous line corresponds to a model in which two power laws, one before and one after a break point, $t_{\mathrm{br}}$, are fit to the data. The model predictions from the power law before $t_{\mathrm{br}}$ have been extrapolated beyond the break (dashed line) to compare with the $18 \mathrm{~cm}$ data which were not used in the fit.
Table 2. Biases in the size determination.

\begin{tabular}{cc|c}
\hline \hline$\xi^{a}$ & Absorption $^{b}$ & $R / X_{\mathrm{OCP}^{c}}$ \\
\hline 0.35 & YES & 0.975 \\
0.30 & YES & 0.970 \\
0.25 & YES & 0.961 \\
\hline 0.35 & NO & 1.012 \\
0.30 & NO & 0.995 \\
0.25 & NO & 0.977 \\
\hline
\end{tabular}

${ }^{a} \xi$ refers to the shell width in units of the source radius (fractional shell width) of the model.

${ }^{b}$ Absorption refers to a blockage of all the emission coming from the part of the shell behind the ejecta (see text).

${ }^{c}$ Ratio of model source size, $R$, to size determined with the CPM, $X_{\mathrm{OCP}}$.

absorption, a smaller $Y_{\mathrm{c}}$ implies a smaller $\rho_{1}$. The ratio $\rho_{2}=\frac{X_{\mathrm{ICP}}}{X_{\mathrm{OCP}}}$ gives an indication of the shell width.

\subsection{Measurements in Fourier space}

In the next section we present our results obtained with the CPM. To compare our results with those of other researchers, obtained using a Fourier analysis, we also analyzed our data in Fourier space. For completeness, we describe the Fourier analysis scheme that we used.

Since the imaginary part of the visibility was always nearly zero, because of the circular symmetry of the source structure and to our choice of the phase center, as explained in Sect. 3, we used only the real part in fitting. The data were weighted using a taper (shown in Fig. 1) to downweight the data from noisy long baselines and avoid any significant change in bias as the source size increased. The model used to fit the data was an optically thin shell with total suppression of the emission from behind the ejecta (see Appendix B). The free parameters in the modelfitting were the source's total flux density, the source's radius and the shell's width. Since in our case the real part of the visibility also has circular symmetry, we azimuthally averaged the data in Fourier space to increase the SNR of the data. The averaging is made using bin sizes that scale inversely with the source size and thus always sample the visibility in the same manner (see Appendix B for details).

A simultaneous fit to source radius and shell width does not usually yield estimates with low uncertainties and low correlations between fitting parameters in cases of low flux density and relatively poor UV-coverage (i.e., $\chi^{2}$ does not then have a sharply defined minimum). In these cases, we fixed one parameter and estimated the other. First, we fixed the shell width (to values that will be given below) and fit the source radius, and later we fixed the source radius to the value obtained with the CPM and fit the shell width. We used the Levenberg-Marquardt (e.g., Gill \& Murray 1978) non-linear least-squares technique, as implemented in the Mathematica 5.0 Package (Wolfram 2003).

\section{Expansion of SN 1993J}

The analysis of all the images of supernova SN 1993J with the CPM yields for the supernova radius the results shown in Table 1. Figure 4 plots those results against time elapsed since the explosion. We also show in Fig. 4 a single fit to both the 3.6 and $6 \mathrm{~cm}$, but not the $18 \mathrm{~cm}$ data. The fit shown was obtained using the fitting procedure implemented in the program Gnuplot (Mathematica gave the same result). Our weighted least squares fit of the supernova radius $R$ as a function of time has 4 parameters since we used the functional form $R \propto t^{m}$, and allowed for two regimes of expansion, each with its own value for $m$. We thus estimated two values of $m$, the epoch of transition between these two regimes ("break-time"), and the radius of the supernova at this break-time. The reduced chi-square of the fit, for the standard errors estimated with the CPM is 0.1 . Hence, we divided each of these standard errors by the square-root of ten to obtain a reduced chi-square of unity. The estimates in Col. 6 of Table 1 are shown with these re-scaled uncertainties. These estimates and standard errors, which in part account for the departures from circularity as explained above, indicate that the radio supernova image remains circularly symmetric over ten years with departures from circularity at the level of $2 \%$ or less (see Appendix A.3 for details). 


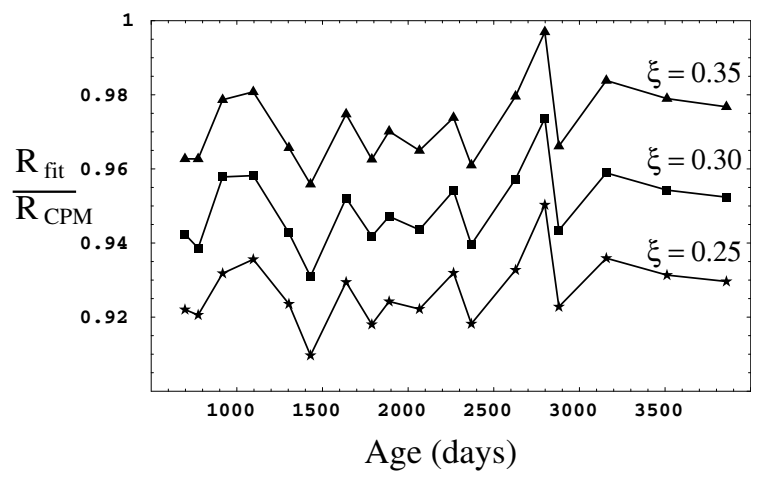

Fig. 5. Comparison of estimates of the supernova radius at $6 \mathrm{~cm}$ obtained using the CPM and a fit to the visibilities with the model explained in the text. The ratio of these estimates is shown for 3 different fractional widths, $\xi$, of the shell model.

The data at $3.6 \mathrm{~cm}$ were only available at early epochs (see Table 1). Thus, it is only in the $6 \mathrm{~cm}$ data that we see a break in the expansion rate. The $3.6 \mathrm{~cm}$ data in Table 1 are consistent with the $6 \mathrm{~cm}$ data. In Fig. 4, the dashed line indicates an extrapolation with the time dependence determined before the break. It is remarkable that the $18 \mathrm{~cm}$ data are consistent with such an expansion in sharp contrast to the data at $6 \mathrm{~cm}$, which require a significantly different expansion rate. The ratio of the sizes at $18 \mathrm{~cm}$ to those at $6 \mathrm{~cm}$ thus evolves after the break as $\propto t^{0.057 \pm 0.016}$. By day 3500 after the explosion, the discrepancy between the size estimate at $6 \mathrm{~cm}$ and $18 \mathrm{~cm}$ is about 0.4 mas, i.e., about $7 \%$.

We also modelled the expansion curve obtained by applying the CPM to the phase-referenced images of the supernova, (i.e., without any self-calibration) and the results obtained are totally compatible with those using self-calibrated images. We obtain $m_{1}=0.845 \pm 0.007, m_{2}=0.799 \pm 0.020$, and $t_{\text {break }}=1500 \pm$ 400. The scatter in the data around the expansion curve and the parameter uncertainties are larger.

The use of size estimates from model fitting to the visibilities (using the model described in Appendix B with the fractional shell width fixed to 0.3 ) also results in fitted parameters which are very similar. In this case, we obtain $m_{1}=0.88 \pm 0.05, m_{2}=$ $0.799 \pm 0.017$, and $t_{\text {break }}=2250 \pm 300$. We notice again that the parameter uncertainties are in this case also larger than those obtained using the CPM with the self-calibrated images.

We repeated this procedure using the values 0.25 and 0.35 for the fractional shell width. The results for the three fractional shell widths are shown in Fig. 5, normalized to the estimates obtained with the CPM. The results shown in the figure are further evidence of the consistency of the results obtained with all methods.

For each value of the shell width of the source model, the ratio $R_{\text {fit }} / R_{\mathrm{CPM}}$ remains rather constant, showing a scatter with a fractional standard deviation of only about $1 \%$. The significance of the different values of the ratio will be discussed in Sect. 6.1. Here, we note only that this constancy in the ratio is tantamount to a replication via fitting of the expansion characteristics determined with the CPM and shown in Fig. 4. We should add that the CPM determines a smoother expansion than the method based on model fitting, since the scatter in the expansion determined with CPM estimates is smaller than with model fitting estimates (the unweighted reduced $\chi^{2}$ of the fit of the supernova expansion using CPM estimates is $17 \%$ lower).
Table 3. Computed ratios $\rho_{1}$ and $\rho_{2}$ (see text) for different shell emission models.

\begin{tabular}{cc|cc}
\hline \hline$\xi^{a}$ & Absorption $^{b}$ & $\rho_{1}$ & $\rho_{2}$ \\
\hline 0.35 & YES & 0.54 & 0.44 \\
0.30 & YES & 0.52 & 0.46 \\
0.25 & YES & 0.52 & 0.47 \\
\hline 0.35 & NO & 0.80 & 0.37 \\
0.30 & NO & 0.78 & 0.39 \\
0.25 & NO & 0.75 & 0.41 \\
\hline
\end{tabular}

${ }^{a}$ Defined in Table 2.

${ }^{b}$ Defined in Table 2.

\section{Emitting region}

\subsection{Central absorption}

Marcaide et al. (1995a,b, 1997, 2005), Bartel et al. (2000, 2002, 2007), Bietenholz et al. (2003, 2005), Alberdi \& Marcaide (2005), and Rupen et al. (1998) argued in favor of the radio emission of SN 1993J originating in a shell. The determination of the details of the emitting shell has been difficult. Bietenholz et al. (2003, 2005) and Marcaide (2005) suggest that the emission appears absorbed in the central part compared to the emission expected from an optically thin shell. Here we present a new data analysis to show that this is indeed the case.

We estimated $X_{\mathrm{ICP}}, X_{\mathrm{OCP}}, Y_{\max }$, and $Y_{\mathrm{c}}$ and, from them, $\rho_{1}$ and $\rho_{2}$ (see Sect. 4.3) from simulated shell emissions as well as from our maps. The shell emission that we used in our simulations are of two types: (1) emission from an optically thin shell; and (2) emission from an optically thin shell with a central absorption that totally blocks the emission from the backside of the shell out to the shell's inner radius. This blockage could be due to absorption of the synchrotron radiation by the ionized ejecta in the line of sight.

In the simulations, we used three values of the fractional shell width. The values were centered on the estimate given by Marcaide et al. (1995a). For each value, we considered the two types of emission described above, namely, with and without central absorption. Table 3 shows the ratios $\rho_{1}$ and $\rho_{2}$ for these simulations. We then determined $\rho_{1}$ and $\rho_{2}$ from our observations. The results are shown in Figs. 6 and 7. We obtain the following mean values: $\rho_{1}=0.51 \pm 0.13$ and $\rho_{2}=0.41 \pm 0.03$ (all uncertainties quoted in this paper are standard deviations.)

A conclusion can be readily drawn from a comparison of these mean values with the values given in Table 3 . The values of $\rho_{1}$ for the models without absorption and with absorption in Table 3 are included in the ranges $0.75-0.80$ and $0.52-0.54$, respectively. The observational result clearly favors the model with absorption and supports the results published earlier.

For estimating $\rho_{1}$ and $\rho_{2}$, we used only maps corresponding to a source size larger than twice the beam of the interferometer to ensure good shell resolution in the maps. For this reason, we did not use the first 5 epochs of Table 1.

\subsection{Shell width}

The shell width can be estimated as explained in Sect. 4.4. To begin, we fixed the source radius to the value determined with the CPM, even though we knew that this value is biased differently depending on the true shell width, as illustrated by the simulations presented in Table 2 . That is, we used the value $X_{\mathrm{OCP}}$ as a first approximation to the value $R$. The estimates obtained for the 


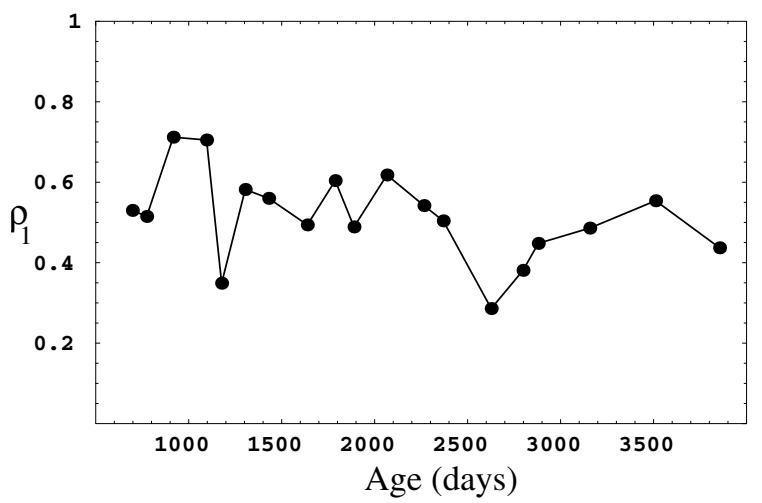

Fig. 6. Estimates of a measure of the central absorption (see text) as a function of time.

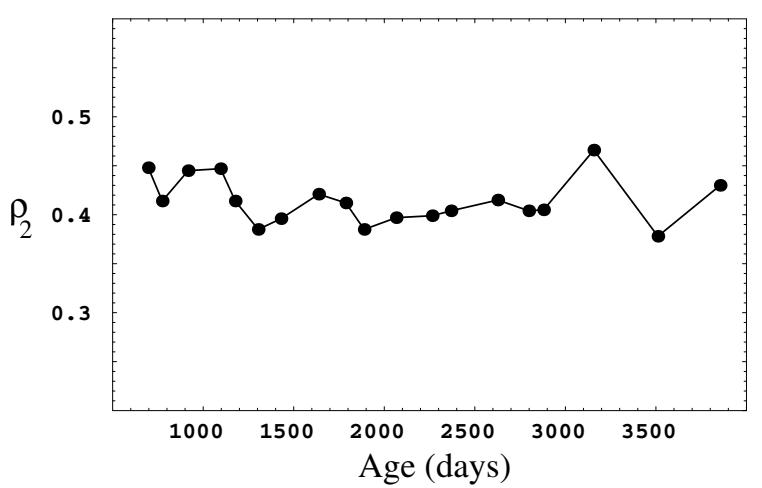

Fig. 7. Estimates of a measure of the fractional shell width (see text) as a function of time.

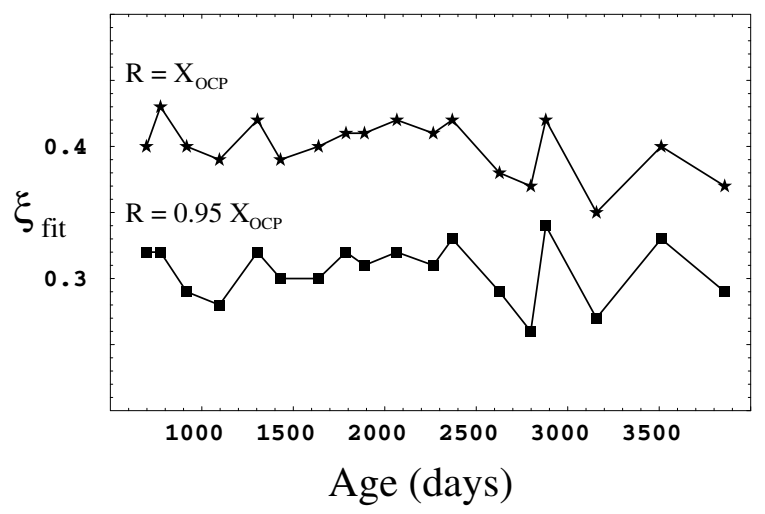

Fig. 8. Fractional shell widths versus supernova age. The widths are determined from $6 \mathrm{~cm}$ data by fitting the visibilities as explained in the text. The source radius is fixed to the value estimated with the CPM (stars) and to $95 \%$ of this value (filled squares).

fractional shell width, $\xi$, were roughly the same for all epochs as shown in Fig. 8. The average estimate is $0.40 \pm 0.04$.

However, to obtain a reliable determination of the fractional shell width we had to know the biases in the determination of the source radius with the CPM to correct for them and to use the corrected $R$ values in the model fitting. For instance, a bias of $5 \%$ in the source radius would translate into a decrease in the fractional shell width from 0.4 to 0.3 , as shown in Fig. 8 .

As we can infer from Fig. 5, estimates of $R_{\mathrm{fit}}$ with the fractional shell width fixed at $0.25,0.30$, and 0.35 , yielded unweighted, average values of the ratio $R_{\mathrm{fit}} / R_{\mathrm{CPM}}$ of $0.93,0.95$, and 0.97 , respectively, with an standard deviation of 0.01 in each case. As mentioned earlier, when we study the bias in the

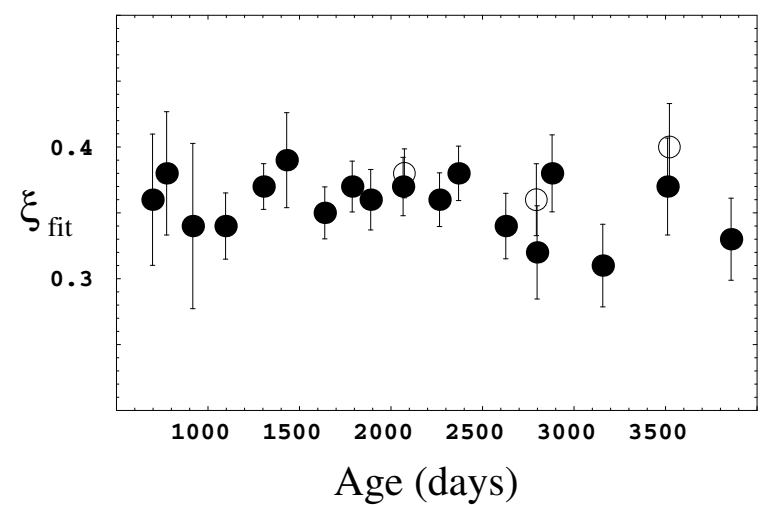

Fig. 9. Fractional shell widths vs. supernova age. These widths are determined from $6 \mathrm{~cm}$ data (filled circles) and $18 \mathrm{~cm}$ data (empty circles) by fitting the visibilities as explained in the text. For consistency (see the text), the source radius is fixed to 0.975 times the value estimated with the CPM.

determination of the source radius with the CPM, using models of different widths, we obtain the results shown in Table 2. By requiring consistency, we find from Table 2 the bias in the model corresponding to the ratio $R_{\mathrm{fit}} / R_{\mathrm{CPM}}$ determined for that same model ( $R$ and $X_{\mathrm{OCP}}$ in Table 2 correspond to $R_{\mathrm{fit}}$ and $R_{\mathrm{CPM}}$, respectively). This consistency in the model with absorption is obtained only for a fractional shell width of 0.35 , which yields a $2.5 \%$ bias and a ratio $R_{\mathrm{fit}} / R_{\mathrm{CPM}}$ of $0.97 \pm 0.01$. For the models with shell widths 0.30 and 0.25 , the corresponding values are $3 \%$ and $0.95 \pm 0.01$, and $4 \%$ and $0.93 \pm 0.01$, respectively. These pairs of values are not as consistent as the pair corresponding to the value 0.35 for the fractional shell width.

We took another redundancy step in this testing: we generated visibility data for conditions similar to the observational ones using a fractional shell width of 0.35 . Then we executed the previous procedures to determine the values of the ratios $R_{\mathrm{fit}} / R_{\mathrm{CPM}}$ using models with fractional shell widths of 0.25 , 0.30 , and 0.35 . The values obtained for the ratios $R_{\text {fit }} / R_{\mathrm{CPM}}$ were $0.94,0.96$, and 0.98 , respectively. In all cases the uncertainty was about 0.01 . These estimates are very close to the values of the ratios that we obtained using the real observations and those same models.

The conclusion seems inescapable: the fractional shell width of a model (with total absorption of emission from the shell region behind the ejecta) most compatible with the data is 0.35 , with the estimate of the outer radius of the shell being about 0.975 times the estimate provided by the CPM. As a final refinement to our determination of the fractional shell width, we re-estimated $\xi$ for all $6 \mathrm{~cm}$ data using as a source radius 0.975 times the CPM estimate. The results are shown in Fig. 9. The weighted mean of these estimates of the fractional width is $0.359 \pm 0.019$. The corresponding results that we obtained for the $18 \mathrm{~cm}$ data are also shown in Fig. 9. In this case, the weighted mean is $0.378 \pm 0.013$, implying that the fractional width might be slightly larger at $18 \mathrm{~cm}$ than at $6 \mathrm{~cm}$.

\section{Discussion of results}

\subsection{Supernova expansion}

In the previous sections, we presented the analysis of our data. To determine the characteristics of the expansion, we estimated the source size for each epoch using two methods: (1) the CPM described in Sect. 4.2, which uses the map of the source in the analysis; and (2) a fit of a model directly to the visibilities. The 
results obtained by the two methods are consistent. However, the CPM is more precise than the fit of a model to the visibilities, and in the latter, one must use an a priori model. In any case, the results are consistent to within $1 \%$ if the bias between the two determinations is taken into account. The determination of this bias, and its constancy in time, is shown in Fig. 5. Thus, the measured expansion rate is the same for both methods. The results, shown in Fig. 4 for the $6 \mathrm{~cm}$ data, are from the use of the CPM. As already discussed in Sect. 5, a break in rate at about day 1500 after explosion can be readily seen in Fig. 4. The expansion index $m$ before that break takes the value $0.845 \pm 0.005$ and after the break $0.788 \pm 0.015$. Remarkably, the $18 \mathrm{~cm}$ data do not follow the $6 \mathrm{~cm}$ data after the break. Rather, the former seem to fall where one would expect for a prediction based on the value of the index before the break.

The $18 \mathrm{~cm}$ data depart significantly from the $6 \mathrm{~cm}$ data. The difference of the expansion indices is $0.057 \pm 0.016$, that is, the ratio of the source size at $18 \mathrm{~cm}$ to the size at $6 \mathrm{~cm}$ evolves in time as $t^{0.057 \pm 0.016}$. A physical model of the source emission should explain this evolution. We propose models for which the $18 \mathrm{~cm}$ data systematically depart from the $6 \mathrm{~cm}$ data. Then, we discuss our results for the shell width.

A straightforward interpretation of these unexpected expansion results states that at the longer wavelength the emitting region extends to the outer shock front in the mini-shell model, while at the shorter wavelength the emitting region is progressively radially smaller and therefore appears to grow at a slower rate than the radius of the outer shock front, this effect becoming discernible after a given epoch, in our case about 1500 days after explosion. In other words, the size of the emitting region should be wavelength dependent. We have attempted to physically model this wavelength dependence; in the process, we eliminated one possible physical explanation but identified two other promising ones.

\subsubsection{Synchrotron mean-life of electrons}

We excluded an explanation based on the mean-life of the emitting electrons. In principle, if electron acceleration occurs near the contact discontinuity, the electrons that emit at $18 \mathrm{~cm}$ $(1.7 \mathrm{GHz})$ should travel further out than the particles that emit at $6 \mathrm{~cm}(5 \mathrm{GHz})$ since their mean-life should be longer. The problem with this explanation is that the mean-life of all of those relativistic electrons is far too long. Using the equations 3.28 and 3.32 from Pacholczyk (1970), we estimate a mean-life of $19 \mathrm{yr}$ for a critical frequency $v_{\mathrm{c}}=5 \mathrm{GHz}(6 \mathrm{~cm}$ wavelength $)$ and a magnetic field $B=0.1$ Gauss (corresponding to a supernova age of 1500 days according to Pérez-Torres et al. 2001). This meanlife is far too long to be compatible with the time needed for the electrons to traverse the shocked circumstellar region, even for random trajectories. Fransson \& Björnsson (1998) proposed a similar mechanism to obtain a wavelength-dependent emitting region. It was based on the mean-life of the emitting electrons, but they assumed that the emission would be generated in the neighborhood of the forward shock. Their mechanism can be discarded on the same grounds as was ours.

\subsubsection{Radially-decreasing magnetic field in the shell}

At present, there is no strong theoretical justification to consider a magnetic field dependence on distance from the constant discontinuity, inside the supernova shell. However, this dependence on distance is plausible, because the field amplification might

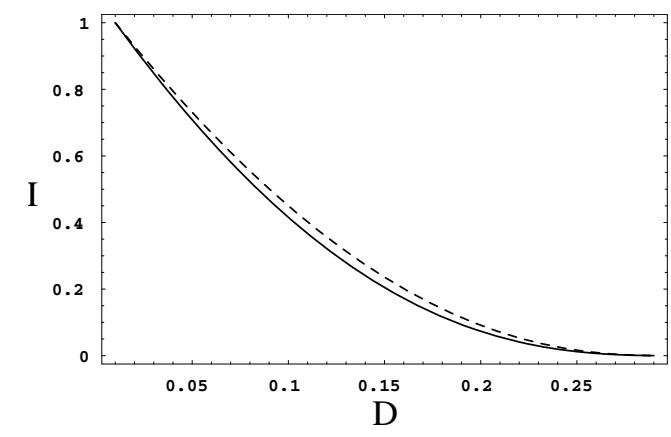

Fig. 10. Radial emission intensity profiles at $6 \mathrm{~cm}$ (solid line) and $18 \mathrm{~cm}$ (dashed line), using a linear radial decrease in the magnetic field and a synchrotron aged electron energy distribution (see text). Each of the profiles is normalized to its corresponding emission at the contact discontinuity. $D$ is the distance from the contact discontinuity in units of the source radius.

take place in the turbulent regime next to the contact discontinuity (e.g., Chevalier \& Blondin 1995). With this motivation, we consider, for example, a linear decrease in the magnetic field with distance from the contact discontinuity and find the observational consequences. Our conclusions will not qualitatively depend on the particular shape of this decrease. Thus, we assume for the magnetic field the expression

$B(D)=B_{\mathrm{c}} \times\left(1-\frac{D}{D_{\max }}\right)$

where $D$ is the distance from the contact discontinuity, $D_{\max }$ is the maximum range of the field, which cannot exceed the distance to the position of the forward shock, and $B_{\mathrm{c}}$ is the magnetic field at the contact discontinuity, which has been chosen such that the average magnetic field over the emitting region is 0.057 Gauss for day 3200, as suggested by Pérez-Torres et al. (2002).

We consider two models, which have in common the essential ingredient of a radially decreasing magnetic field. The first one concerns synchrotron aging of the emitting electrons and the second the finite sensitivity of the interferometers. We describe each of them in turn.

Synchrotron aging translates into a deviation of the electron energy distribution from the canonical $N \propto E^{-p}$ distribution at high energies. Chandra et al. (2004) suggested synchrotron aging in SN 1993J, based on their observed radio spectrum. We should note however that this suggestion is not supported by the work of Weiler et al. (2007).

If we assume emission within an optically thin medium, we can compute for a synchrotron-aged electron population (see Appendix C) the 2D image corresponding to the emission profile in Fig. 10 for each of the radio wavelengths. For each wavelength, the profile corresponding to the azimuthal average of the 2D image, convolved with a Gaussian beam, is shown in Fig. 11. We apply the CPM (see Sect. 4.2) to estimate the size of each image.

Clearly, as in Fig. 10, the profile reaches a given level of intensity further out at the longer wavelength, thus increasing the size estimate of the CPM by about $0.5 \%$ over the corresponding estimate at the shorter wavelength. This increase falls short of the observational result by a factor of 4 , but it does go in the right direction. A steeper radial drop in the magnetic field would decrease the shortfall. For example, an exponential drop would decrease it by about a factor of 2 . A possible high-energy cutoff in the relativistic electron distribution would also contribute in 


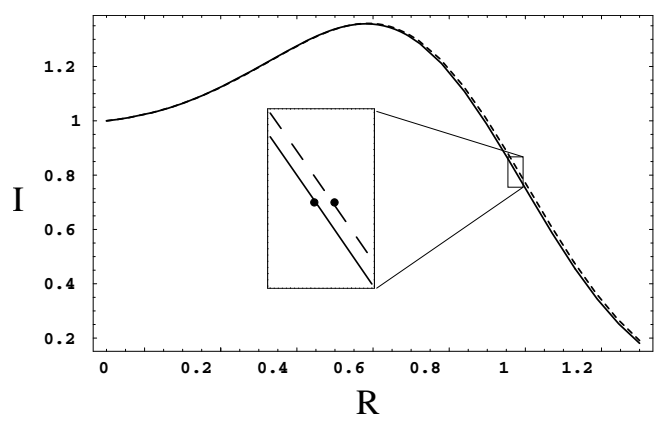

Fig. 11. Profiles of the azimuthal averages of the maps obtained from the radial intensity distributions shown in Fig. 10 normalized to the corresponding intensity at the source center. The units for the $x$-axis are normalized to the source size. As in the previous figure, the solid line corresponds to $6 \mathrm{~cm}$ and the dashed line to $18 \mathrm{~cm}$. The dots shown in the enlargement correspond to the outer common point of each profile.

the same direction to yield a size estimate larger at $18 \mathrm{~cm}$ than at $6 \mathrm{~cm}$.

A consequence of the previous explanation is that there should also be a difference in the size estimations between 6 and $3.6 \mathrm{~cm}$, although the difference should be smaller than that between 18 and $6 \mathrm{~cm}$ (see Eq. (C.3) in Appendix C). We do not find such a difference in our own data. However, our data at $3.6 \mathrm{~cm}$ are restricted to the earliest epochs and do not overlap temporally with the data at $6 \mathrm{~cm}$.

The limited sensitivity of an interferometric array enhances considerably the difference of the source size at 6 and $18 \mathrm{~cm}$, if measurable. Indeed, as shown in Fig. 12b, which is an enlargement of the outer region of Fig. 10 but where both types of emission are normalized to the $6 \mathrm{~cm}$ emission at the contact discontinuity, the intersection of these curves with a realistic noise level (i.e., obtained in the simulation using typical antenna system temperatures) takes place at a quite different radial position at $6 \mathrm{~cm}$ than at $18 \mathrm{~cm}$ for a linear radial decrease in the magnetic field, but not for a model with a constant magnetic field (Fig. 12a). Both intersections also occur at smaller values of $D$ for Fig. 12b than for Fig. 12a.

As indicated in Fig. 12a, for a constant magnetic field the realistic noise intersection for both wavelengths occurs at practically the same value of D. Hence, the measurement of the source radii in the maps corresponding to those source emission intensity profiles would be practically unaffected by the noise. (Note that the profiles at 6 and $18 \mathrm{~cm}$ shown in Fig. 12b are the same as in Fig. 10, but appear to differ only because the emission at both wavelengths was normalized to the $6 \mathrm{~cm}$ emission level. For the model considered, the source emission is stronger at $18 \mathrm{~cm}$ than at $6 \mathrm{~cm}$.) For unrealistically large noise levels (i.e., a large fraction of the source flux density per beam), a difference in the sizes at the two wavelengths would in principle also be noticeable, but in practice at those noise levels we would not even be able to reconstruct the VLBI maps with sufficient quality to detect the effect we discuss here.

For a magnetic field that decreases radially (Fig. 12b), the source emission above the noise extends to a larger $D$ at $18 \mathrm{~cm}$ than at $6 \mathrm{~cm}$. Therefore, the source radius would also be smaller at $6 \mathrm{~cm}$ than at $18 \mathrm{~cm}$. The difference in the size estimates between 6 and $18 \mathrm{~cm}$ at day 3200 (see previous subsection) is about $2 \%$ and has the "right" sign.

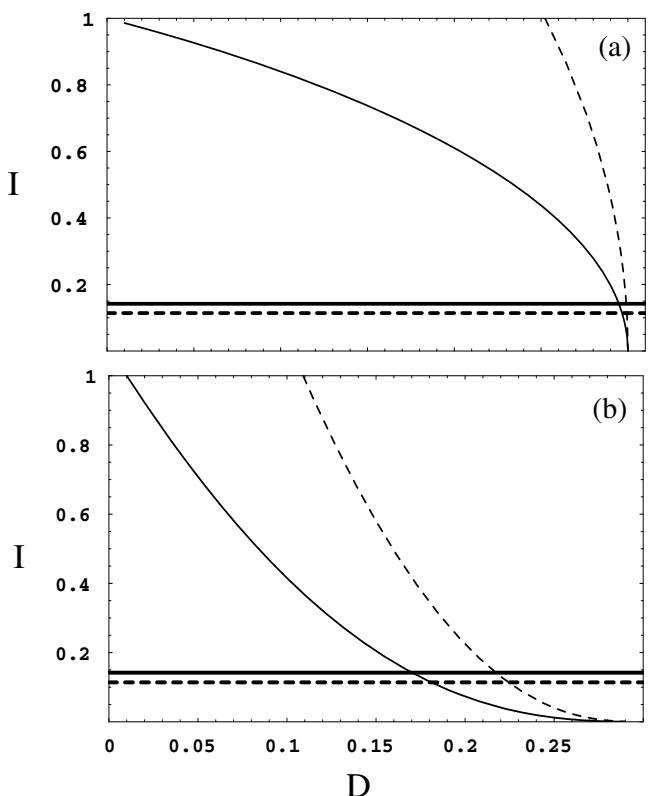

Fig. 12. Radial emission intensity profiles at $6 \mathrm{~cm}$ (solid line) and $18 \mathrm{~cm}$ (dashed line) normalized to the emission at $6 \mathrm{~cm}$ at the contact discontinuity. The horizontal lines $(6 \mathrm{~cm}$, thick solid line; $18 \mathrm{~cm}$, thick dashed line) indicate realistic noise levels: a) for a constant magnetic field in the emitting region; and b) for a linear radial decrease in the magnetic field (see Eq. (1)). A synchrotron-aged electron energy distribution (see Eq. (C.4)) was used in both cases. $D$ is the distance from the contact discontinuity in units of the source radius.

Table 4. Spectral indices, $\alpha$, determined from our VLA data for a subset of observations for which we have quasi-simultaneous 6 and $18 \mathrm{~cm}$ observations (M 81 used as calibrator).

\begin{tabular}{c|c}
\hline \hline Age (days) & $\alpha$ \\
\hline 2794 & $0.68 \pm 0.01$ \\
$2820^{a}$ & $0.67 \pm 0.02$ \\
3511 & $0.555 \pm 0.010$ \\
3858 & $0.50 \pm 0.04$ \\
\hline
\end{tabular}

${ }^{a}$ From Pérez-Torres et al. (2002).

\subsubsection{Changes in the opacity of the ejecta}

The effects considered in the previous sections may account for some of the differences in the expansions observed at 6 and $18 \mathrm{~cm}$. However, these effects seem to be insufficient to account for a $4 \%$ difference in the sizes at day 3200 or for a larger difference at later epochs. On the other hand, flux density measurements, made with the VLA at epochs where we have data at both 6 and $18 \mathrm{~cm}$, also show a trend that is worthwhile discussing. Fransson \& Björnsson (1998) suggested that for late epochs (beyond day 1000 after explosion) the spectral index between 6 and $18 \mathrm{~cm}$ should remain rather constant, $\alpha \sim 0.75$ $\left(S_{v} \sim v^{-\alpha}\right)$. However, observationally the spectral index of the supernova does not remain constant but decreases with time (see our Table 4 and Fig. 3a of Weiler et al. 2007). How can we explain this change?

In Fig. 4, we plot the 6 and $18 \mathrm{~cm}$ flux densities obtained from our VLBI maps and given in Table 1 (M 81 was used as flux density calibrator; our estimates may thus differ systematically from those of Weiler et al. 2007). We notice a sharp change in the evolution of the $6 \mathrm{~cm}$ map flux density after day 1500 , which correlates with the change in slope of the expansion measured at that same wavelength. This correlation may have some 


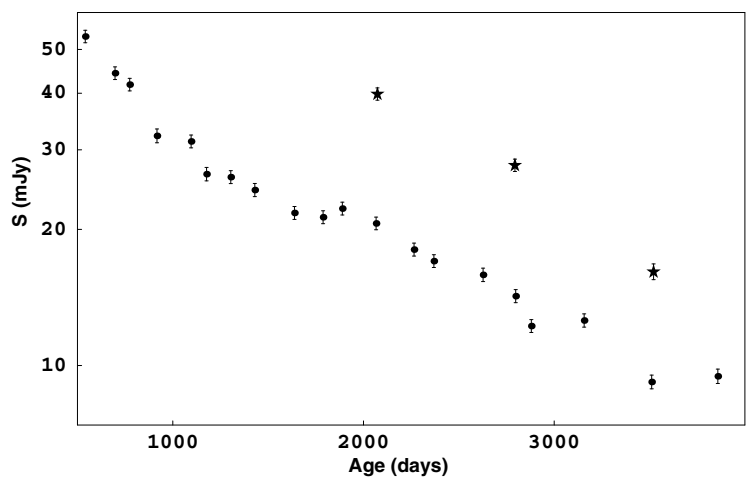

Fig. 13. Time evolution in the total map flux densities of SN 1993J, as determined from the VLBI data. See Table 1 for the values. The standard deviations were estimated by adding 3 times the rms map noise to $3 \%$ of the total flux density, to account for calibration systematics. Circles and stars correspond to 6 and $18 \mathrm{~cm}$ data, respectively. Weiler et al. (2007) presented similar light curves observed with the VLA. The bump is also very conspicuous in their curves at several wavelengths.

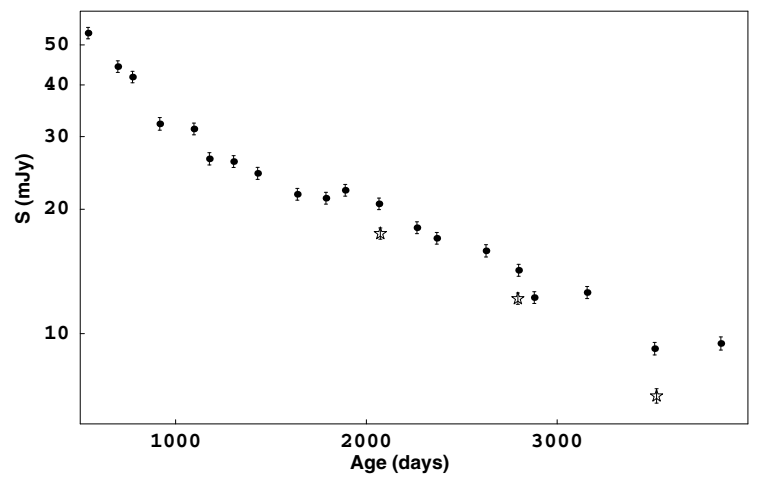

Fig. 14. Same as previous figure, but with the $18 \mathrm{~cm}$ data converted (empty stars) to a $6 \mathrm{~cm}$ equivalent total flux density using a spectral index of value 0.75 .

significance. The evolution in the $6 \mathrm{~cm}$ flux density in Fig. 4 appears to exhibit an increase with respect to the evolution expected from previous epochs. A natural way of obtaining this increase could be to start receiving emission from a region of the shell that was suppressed at previous epochs, namely, the emission from the side of the shell behind the ejecta. An opacity of the ejecta that decreases with time making them more transparent to $6 \mathrm{~cm}$ than to $18 \mathrm{~cm}$ radiation is sufficient.

In spite of what has been said above, we transform the $18 \mathrm{~cm}$ flux densities into "hypothetical $6 \mathrm{~cm}$ flux densities" using the spectral index suggested by Fransson \& Björnsson (1998) and compare them with the true $6 \mathrm{~cm}$ flux densities. Surprisingly, as shown in Fig. 14, transformed $18 \mathrm{~cm}$ flux densities follow the $6 \mathrm{~cm}$ flux-density evolution expected from epochs earlier than day 1500 . Hence, a possible interpretation might be the following: the true spectral index is $\sim 0.75$, as suggested by Fransson \& Björnsson (1998), or close to it (Weiler et al. 2007), and the spectral index evolution we observed is due solely to an increase in detected emission at $6 \mathrm{~cm}$ from the shell region behind the ejecta because the latter becomes more transparent at $6 \mathrm{~cm}$ than at $18 \mathrm{~cm}$ after day 1500 .

As noticed in an earlier section and quantified in Table 2, a change in the emission-absorption model will also result in a different estimate of the source size for a given set of data. Thus, as shown in Table 2, for a given data set a model with absorption will yield a larger estimate of the source size than a model without absorption. The difference in the estimates can be as large as $2.5 \%$ for a change in opacity from total absorption to no absorption at all. For a shell model (with a shell of 0.30 fractional width) of radius $R$ with total absorption, the size determined by the CPM would be $1.03 R$. For the same model of radius $R$ without absorption, the size determined by the CPM would instead be only $1.005 R$.

Thus, a transition from a model with absorption to one with no-absorption would result in a decrease in the size estimated, if either the CPM or fitting to the visibilities is used. Were the transition (decrease in opacity) to take place over a period of time, the net effect would be a progressive decrease in size estimates during this period, resulting in turn in a decrease in the estimate of the deceleration parameter (namely, the estimated $m$ would be smaller than the true $m$ because of the decrease in the absorption in the source). Consequently, after the ejecta become fully transparent to the $6 \mathrm{~cm}$ radiation, the true deceleration parameter $m$ will be recovered. Unfortunately, given the flux density evolution in the source (Weiler et al. 2007), this recovery will be unlikely to take place while SN 1993J can still be mapped at $6 \mathrm{~cm}$.

\subsection{Shell width}

We determined the shell width as a fraction of source radius, using for the latter the bias-corrected results from the CPM and model fitting to the visibilities as explained in Sect. 6.2. The results given in Fig. 9 show a rather similar fractional width determination for all epochs. The average values of the 6 and $18 \mathrm{~cm}$ results infer fractional shell width values of $0.359 \pm 0.019$ and $0.378 \pm 0.013$, respectively.

These previous estimates were based on a model that assumed total absorption of the emission from the back of the shell (behind the ejecta). Partial absorption is clearly also compatible with our data and would yield a smaller fractional value for the shell width. In this sense, the result given above is an upper bound to the fractional shell width.

We note that the uncertainties (and scatter) in the shell width estimates from the $6 \mathrm{~cm}$ data for the supernova age range of 1500-2500 days, as shown in Fig. 9, are generally smaller than for earlier and later ages. For earlier epochs, the supernova size is smaller and the determination more difficult, whereas for later epochs the flux density of the source is lower and hence the data are relatively noisier. The $6 \mathrm{~cm}$ data for the range 1500-2500 days (corresponding to 6 epochs of observation) are optimal, in a sense, for the determination of the shell width. Thus, we used these data to simultaneously determine the source size, the shell width, and the degree of absorption of the model. These data suffice to reliably obtain the estimates of all 3 parameters. The results are shown in Table 5. The average estimate of the fractional shell width is $0.31 \pm 0.04$ and the degree of absorption in the corresponding model is $(80 \pm 14) \%$. The large uncertainties are caused mostly by the first two epochs. (Using only the last four epochs, we obtain $0.33 \pm 0.04$ and $(73 \pm 7) \%$, respectively.) The ratio $R_{\mathrm{fit}} / X_{\mathrm{OCP}}$ is $0.97 \pm 0.03$, which is consistent with the theoretical bias expected for a model with $80 \%$ absorption and a fractional shell width of 30\%: 0.968. As expected, less absorption translates into a smaller shell width estimate.

In view of the previous results about absorption, we reestimated the fractional shell width using data from all epochs, as before, but now for a model with a fixed absorption of $80 \%$. After taking into account the corresponding bias of the CPM, 0.968, we present the results in Fig. 15. This figure contains our most accurate estimates of the fractional shell widths. The average values for the $6 \mathrm{~cm}$ and $18 \mathrm{~cm}$ results are $0.31 \pm 0.02$ 
Table 5. Model fitting results for epochs between 1500 and 2500 days.

\begin{tabular}{c|ccc}
\hline \hline $\begin{array}{c}\text { Age } \\
\text { (days) }\end{array}$ & $R_{\mathrm{fit}} / X_{\mathrm{OCP}}{ }^{a}$ & $\xi_{\text {fit }}{ }^{b}$ & $\begin{array}{c}\text { Absorption }^{c} \\
(\%)\end{array}$ \\
\hline 1638 & $0.935 \pm 0.016$ & $0.24 \pm 0.03$ & $108 \pm 4$ \\
1788 & $0.952 \pm 0.010$ & $0.294 \pm 0.017$ & $91 \pm 5$ \\
1889 & $1.009 \pm 0.013$ & $0.374 \pm 0.013$ & $77 \pm 2$ \\
2066 & $0.987 \pm 0.013$ & $0.315 \pm 0.019$ & $61 \pm 5$ \\
2265 & $1.000 \pm 0.012$ & $0.355 \pm 0.017$ & $80 \pm 6$ \\
2369 & $0.960 \pm 0.012$ & $0.27 \pm 0.02$ & $67 \pm 6$ \\
\hline
\end{tabular}

${ }^{a}$ Fitted source radius, normalized to the CPM estimate.

${ }^{b}$ Fitted fractional shell width.

${ }^{c}$ Fitted percentage of absorption by the ejecta.

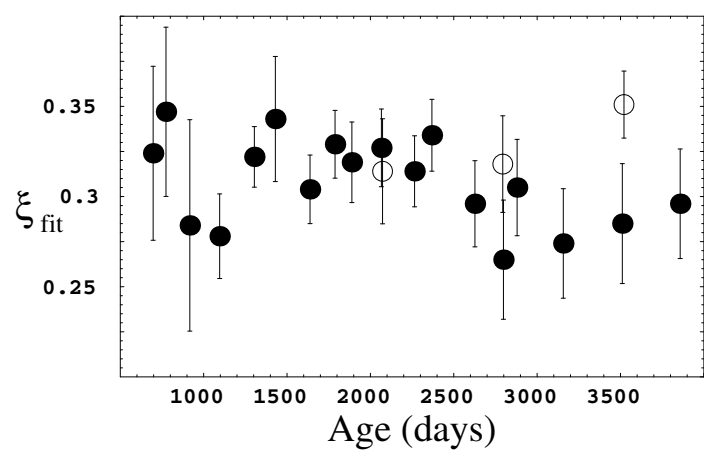

Fig. 15. Relative shell widths versus supernova age for a model with $80 \%$ absorption. The widths are determined from $6 \mathrm{~cm}$ (filled circles) and $18 \mathrm{~cm}$ data (empty circles) by model-fitting the visibilities. For consistency (see text), the source radius is fixed at 0.968 times the value estimated with the CPM.

and $0.335 \pm 0.017$, respectively. The degree of absorption at $18 \mathrm{~cm}$ cannot be determined from our data; it might well be $100 \%$, in which case the estimate of the fractional shell width at this wavelength would be $0.378 \pm 0.013$.

Figure 15 hints at a possible decrease in the fractional shell width at $6 \mathrm{~cm}$ for a supernova age beyond day 2500, while the corresponding estimates for $18 \mathrm{~cm}$ seem to show, if anything, the opposite trend. We expect this kind of evolution for each of the 3 mechanisms proposed in the previous section to explain the characteristics of the expansion at $6 \mathrm{~cm}$ :

1. synchrotron aging would reduce the extent of the emitting region and hence the source size (outer radius of the emitting region). Given that the inner radius would be unchanged by this mechanism, the fractional shell width would decrease. This mechanism would account for about a $0.5-1 \%$ decrease in the source size (1.5-3\% in the shell width);

2. a decreasing magnetic field with radial distance, coupled with the limited sensitivity of the interferometers would account for a decrease in the source size and, hence, by the same argument as for mechanism (1), of the shell width. This mechanism would account for about a $2 \%$ decrease in the source size (6\% in the shell width). Mechanism (1) would correspond to a real decrease, mechanism (2) to a decrease caused by an instrumental effect. In both cases, a radially decreasing magnetic field is necessary;

3. a changing opacity translates into changing source size estimates when a fixed source model is used in the size estimation process. Part of the enhanced deceleration beyond day 1500 , apparently seen at $6 \mathrm{~cm}$, might be caused by use of a model with fixed absorption. Decreasing absorption would thus lead to underestimates of the source size. Since in fitting the shell width, the source size is a fixed parameter, an underestimate of the source size would translate into an underestimate of the shell width. This mechanism would account for a maximum of $2.5 \%$ (change from totally opaque to totally transparent ejecta at $6 \mathrm{~cm}$ ) in the source size (up to $7.5 \%$ of the shell width). However, since the absorption is apparently not lower than about $80 \%$, this mechanism could account for only a fraction of the previous estimates.

\section{Comparison with other VLBI results}

\subsection{Supernova expansion}

Bartel et al. (2002) reported VLBI observations of SN 1993J made at several wavelengths over a similar time range as we now report. Their analysis of the VLBI data was carried out with different tools than ours. They estimated the source size by fitting models directly to the visibilities using a modified version of task UVFIT of the NRAO AIPS Package. The model used in those fits was a three-dimensional spherical shell of uniform volume emissivity and fractional shell width of 0.2 . They estimated the outer radius of the shell and also the plane-of-thesky coordinates of its center with respect to the phase center of the supernova. In a previous paper, Bietenholz et al. (2001) concluded that these center coordinates remain fixed (within the uncertainties) with respect to the core of M 81; thus, it is unclear to us why they were included as free parameters (presumably as a check on their previous work). Including these parameters is equivalent to determining the slopes of the imaginary part of the visibilities. Because of the remarkable circular symmetry of the source, these imaginary parts contain no significant structure information, especially those for the short baselines that are the most relevant to the fits.

As explained earlier in this paper, we determined the shell outer radius with two methods. For each, we assumed the same center for all epochs and found that, to within the noise of the measurements, the imaginary part of the visibilities vanished and so we could fit the outer shell radius using only the real part. As a consequence, we estimated fewer parameters in the fit and, perhaps for that reason, the solutions are more stable in our case than in Bartel et al. (2002). We also used three-dimensional spherical shells of uniform emissivity, but including absorption by the supernova ejecta. Hence, Bartel et al.'s results are not directly comparable to ours.

Bartel et al. (2002) determined a deceleration parameter for each of four supernova age ranges (see their Table 4), noting that "the four time intervals with different decelerations can be distinguished by eye." For each of the four age ranges, Bartel et al. determined a deceleration for each observed wavelength and noticed differences in the determinations. They also considered decelerations obtained from the combined data set as being representative.

Based on their determination of the deceleration for each of the four supernova age ranges, Bartel et al. inferred an increasing deceleration of the expansion followed at about day 1893 by a slowing deceleration. This evolution in the deceleration, they stated, provides support to the predictions made by Mioduszewski et al. (2001) from their hydrodynamic simulations. Those simulations depend very strongly on certain features of the density profile of the ejecta (explosion model 4H47 provided by Nomoto's group), which are of some concern. Simulations with less specific -and more accepted- explosion models would not have produced these results. Hence, a 
re-analysis of the expansion data provided by Bartel et al. (2002) might be of interest. A re-analysis of all VLBI data from Bartel's group was part of the Ph.D. thesis of Martí-Vidal (2008) and will be published elsewhere.

We fit the expansion model that we described in Sect. 5 to the data published by Bartel et al. (2002). This expansion model considers two deceleration regimes and the time of change between them, yielding three parameters to be estimated. At $6 \mathrm{~cm}$, we used 20 outer-shell radius determinations from Bartel et al. (2002), which ranged from day 223 through day 2996, and obtained the deceleration parameter estimates of $m_{1}=0.85 \pm 0.03$ and $m_{2}=0.78 \pm 0.04$, with a break at day $900 \pm 500$. At $3.6 \mathrm{~cm}$, the 26 data points, which extend from day 50 to day 2787, yield the estimates $m_{1}=0.96 \pm 0.04$ and $m_{2}=0.80 \pm 0.02$ with a break at day $320 \pm 90$. However, if we ignore the $3.6 \mathrm{~cm}$ data from before day 223, we obtain $m_{1}=0.84 \pm 0.04$ and $m_{2}=0.78 \pm 0.04$ with the break at day $700 \pm 600$. In all cases, the uncertainties given are scaled such that the reduced chi-squares of the fits are unity. Given the uncertainties shown, the parameter estimates for the 6 and $3.6 \mathrm{~cm}$ data for days after 223 are remarkably similar.

These estimates of the deceleration parameters are also very similar to those we obtained from our own data using what we think is a more accurate method, the CPM: $0.845 \pm 0.005$ and $0.788 \pm 0.015$, although we find the break time to be at day $1500 \pm 300$ in our case. Thus, we conclude that the Bartel et al. data are compatible with just one change in deceleration and that there is no need to invoke changes in deceleration at other times. The agreement of the results given by Bartel et al. with the hydrodynamic model of Mioduszewski et al. may therefore not be significant.

Bartel et al. (2002) also published estimates of the source size for 8 epochs at $13 \mathrm{~cm}$ and for 3 epochs at $18 \mathrm{~cm}$. The $13 \mathrm{~cm}$ estimates are consistent with those for 3.6 and $6 \mathrm{~cm}$ for the first 1000 days but for later epochs the estimates are systematically larger than for $6 \mathrm{~cm}$. Except for their estimate around day 1000, those results are consistent with our findings, to within the estimated standard errors. However, the $13 \mathrm{~cm}$ data do not show a clear trend of departure from the $6 \mathrm{~cm}$ curve. The Bartel et al. $18 \mathrm{~cm}$ estimates are also larger than the $6 \mathrm{~cm}$ estimates for the same epochs, but have significant scatter. We reanalyzed the VLBI observations from Bartel's group for days $1692(6 \mathrm{~cm})$ and $3164(18 \mathrm{~cm})$. These size determinations are consistent with our determinations from our own data and indicate that Bartel et al.'s estimate at $18 \mathrm{~cm}$ is an underestimate ( 4\%). In any case, since the $18 \mathrm{~cm}$ estimates are larger than those at $6 \mathrm{~cm}$ at the latest epochs, those authors (see Fig. 6 in Bartel et al. 2002) interpreted this as slowing deceleration. Even the last $6 \mathrm{~cm}$ estimates of Bartel et al. are probably overestimates, if the true shell width decreases in that period, as we concluded in Sect. 7. Keeping a model with a fixed fractional shell width, with no absorption, in fitting data corresponding to a decreasing shell width, or monotonically decreasing absorption by the ejecta, leads to a progressively increasing overestimate of the size. This overestimation of the sizes from the $6 \mathrm{~cm}$ data has two consequences: it reinforces one's impression of a slowing deceleration and prevents one from discerning the progressively increasing difference in the sizes at 6 and $18 \mathrm{~cm}$.

\subsection{Shell width}

Bartel et al. (2002) required their data to meet a set of criteria before using them to estimate the shell width. A total of 16 epochs fulfilled their criteria ( 10 at $3.6 \mathrm{~cm}$ and and 6 at $6 \mathrm{~cm}$ ). They estimated a fractional shell width of $0.25 \pm 0.02$ by fitting a spherical shell model, without any absorption, to their data. They deferred to a later article by Bietenholz et al. (2003) discussion of adding (possible) absorption in their model. In the latter paper, the authors estimated the fractional shell width as $0.25 \pm 0.03$, using a simple disk model to simulate a $25 \%$ absorption of the radiation from the central part of the source. With such a small amount of absorption, the estimate of the fractional shell width is about the same as with a model without absorption. They mentioned that using a larger disk model and a stronger absorption the relative shell width could be as large as 0.35 , but they excluded this option because they considered the fit to the data to be worse. In our opinion, the disk model they used to simulate the absorption was too restrictive and directly caused their inadequate fit (see their Fig. 13).

\section{Comparison with results from optical observations}

Fransson et al. (2005) presented HST ultraviolet spectra from the nebular phase of the expansion of SN 1993J (days 670-2585). They found that the spectrum remained remarkably constant in time. However, they identified small temporal changes in the shape of the Mg II line, which, as mentioned by these authors, changes in concordance with the shape of the $\mathrm{H}_{\alpha}$ line observed by Matheson et al. (2000a). Since the changes between days 1063 and 1399 are small, Fransson et al. (2005) averaged the spectra from those epochs to increase the signal-to-noise ratio, and fit these data and the $\mathrm{H}_{\alpha}$ line data on day 976 to a model with a constant emissivity shell of inner velocity $V_{\text {in }}$ and outer velocity $V_{\text {out }}$. As shown in their Fig. 3, a combination of $V_{\text {in }}=7000$ and $V_{\text {out }}=10000 \mathrm{~km} \mathrm{~s}^{-1}$ fits the boxlike data rather well. As good a fit can be obtained either with a rather thin shell, regardless of the emissivity structure, or with a thicker shell of constant emissivity. There are also indications that $V_{\text {in }}=6000 \mathrm{~km} \mathrm{~s}^{-1}$ could also fit the lines (see the discussion in Fransson et al. 2005). Thus, one should take the $7000 \mathrm{~km} \mathrm{~s}^{-1}$ as an upper bound to the lowest velocities in the optically emitting shell. For an homologous expansion, these velocity measurements would translate into a $30 \%$ wide shell.

How does this optically emitting shell relate to the radio emitting shell? For the same epochs, it is rather remarkable that the upper and lower velocities in the optical and radio shells are nearly the same. By estimating the velocity of the outer radio surface from the expansion shown in Fig. 4, and assuming the distance of $3.63 \pm 0.34 \mathrm{Mpc}$ to M 81 (Freedman et al. 1994) as the distance to SN 1993J, we derive the range 10000-10500 $\mathrm{km} \mathrm{s}^{-1}$ for days 1063-1399, which implies velocities in the range $6900-7250 \mathrm{~km} \mathrm{~s}^{-1}$ in the inner radio surface, for our determination of the fractional shell width, which remains nearly constant at $0.31 \pm 0.04$ (i.e., the shell expands self-similarly). The optical emission, instead, is thought to originate in a cool dense shell in the shocked ejecta (e.g., Fransson 1984). This shell is necessarily spatially thin. Consequently, the optically emitting shell cannot have an homologous structure. The problem with this optical-radio comparison is that the inner part of the radio emitting shell, next to the contact discontinuity, is further from the center than is the optically emitting shell, but at lower velocities than most of the shell material emitting in the optical. Were these optical velocities to be expansion velocities, would the optical shell eventually enter the radio shell?

Only for the optical emission taking place at the contact discontinuity would the velocity of the optical lines be equal to the expansion velocity of the contact discontinuity. In that case, the optical velocities and the velocities inferred from VLBI would 


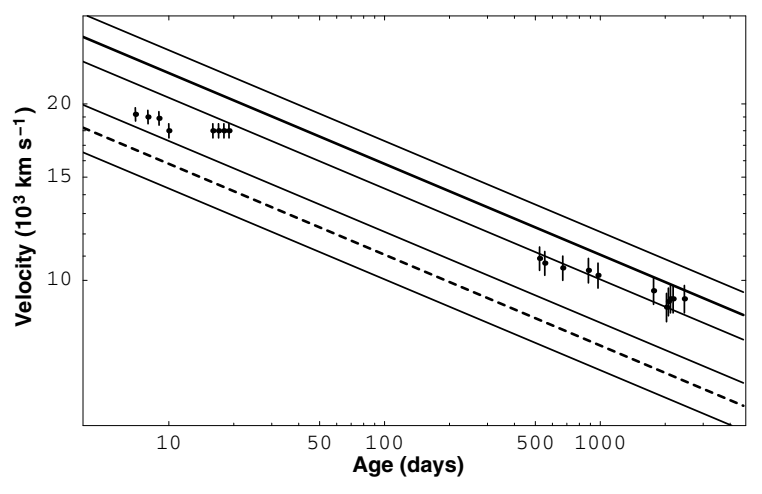

Fig. 16. Velocities of the inner (dashed line) and outer (solid line) radio shell surfaces computed from our expansion model using $m=0.845$ and a distance of $3.63 \pm 0.34 \mathrm{Mpc}$ to SN 1993J. The thin lines at each side of the dashed and solid lines indicate the uncertainties. Filled circles (and error bars) are the maximum velocities of the $\mathrm{H} \alpha$ line (and their standard deviations) reported by various authors (see text).

be directly comparable. In Fig. 16, we show the velocities of the inner and outer radio shell surfaces (each with the corresponding uncertainty) as computed from our expansion model using $m=0.845$. In that figure, we also plot the maximum velocities at the blue edge of the $\mathrm{H} \alpha$ line reported by Trammell et al. (1993), Lewis et al. (1994), Finn et al. (1995), and Matheson et al. (2000b), all as given in Bartel et al. (2007). It is interesting to see that optical velocities appear closer to the inner shell surface velocity for early days and further from it at late days, thus indicating a lower deceleration of the regions responsible for the optical emission than that of the radio shell. In other words, if this high velocity optical emission takes place at the tips of the Rayleigh-Taylor fingers (a kind of effective contact discontinuity) the comparison of the velocities is indicating a progressive penetration of the fingers into the shocked circumstellar medium. Bartel et al. (2007) also arrived at a similar conclusion. Chevalier \& Blondin (1995) proposed this kind of evolution in the Rayleigh-Taylor fingers from hydrodynamical simulations.

\section{Conclusions}

We have studied the growth of the shell-like radio structure of supernova SN 1993J in M 81 from September 1993 to October 2003 with very-long-baseline interferometry (VLBI) observations at the wavelengths of $3.6,6$, and $18 \mathrm{~cm}$. We used two methods to analyze our data: a new method, named the Common Point Method (CPM), described in detail in Sect. 4.2 and Appendix A of this paper; and by fitting a specific parameterized model to the visibilities, as described in Appendix B. The CPM allows us to accurately estimate the source size on the sky plane for a (nearly) circularly symmetric compact radio structure such as SN 1993J. The model fitting estimate instead depends strongly on the source model used in the fitting. Thus, changes in the emission structure of the supernova during its evolution could affect the model fitting estimates of the source size, and hence the determination of the supernova expansion, especially if a time-independent model were used.

The source structure remains circularly symmetric (with deviations from circularity of less than $2 \%$ over almost 4000 days). Using our data at 3.6 and $6 \mathrm{~cm}$, we can characterize the decelerated expansion of SN 1993J until day 1500 after explosion with a single expansion parameter $m=0.845 \pm 0.005\left(R \propto t^{m}\right)$. However, from that day onwards the expansion appears different when observed at 6 and $18 \mathrm{~cm}$. At the latter wavelength, the expansion can indeed be characterized well by the same $m$ as before day 1500 (self-similar expansion), while at the former wavelength the expansion appears more decelerated and is characterized by another expansion parameter, $m_{6}=0.788 \pm 0.015$. From about day 1500 onwards, the radio source size is progressively smaller at $6 \mathrm{~cm}$ than at $18 \mathrm{~cm}$.

Our interpretation is that the expansion of the supernova is self-similar and characterized by a single expansion parameter, $m=0.845 \pm 0.005$, which applies to all data before day 1500 after explosion and to only $18 \mathrm{~cm}$ data all the time. In our interpretation, the $6 \mathrm{~cm}$ results would not represent the advance of the supernova radius because at $6 \mathrm{~cm}$ the size measured would be systematically smaller than the source size. We can think of several ways in which this could happen:

- Changing opacity affecting the emission at $6 \mathrm{~cm}$ from the part of the shell behind the supernova ejecta. Before about day 1500 after explosion, the supernova ejecta would be opaque to the radiation at all wavelengths but from then onwards the opacity at $6 \mathrm{~cm}$ would start to decrease, while remaining unchanged at $18 \mathrm{~cm}$. This hypothesis receives additional support from the evolution in the total flux density at $6 \mathrm{~cm}$.

- Radially decreasing magnetic field in the emitting region. The consequences of the radially decreasing magnetic field would come either from the limited sensitivity of the radio interferometers or from synchrotron aging, or from both. In the first case, emission from the outer regions detectable at $18 \mathrm{~cm}$ would fall below the detection limit at $6 \mathrm{~cm}$. In the second case, the emission structure would be wavelength dependent.

Combining the two methods, i.e., CPM and model fitting, we determined from $6 \mathrm{~cm}$ data a fractional width of the radio shell of $31( \pm 2) \%$, and a degree of absorption of the radio emission from the backside of the shell behind the ejecta of $80 \%$. Unfortunately, we can determine from our data neither a possible evolution in the absorption at $6 \mathrm{~cm}$ nor the degree of absorption at $18 \mathrm{~cm}$. For $80 \%$ absorption at $18 \mathrm{~cm}$, we estimate a fractional shell width at $18 \mathrm{~cm}$ of $33.5( \pm 1.7) \%$. For $100 \%$ absorption, the fractional shell would be somewhat larger: $37.8( \pm 1.3) \%$. In both cases, the shell at $18 \mathrm{~cm}$ is expected to be thicker than at $6 \mathrm{~cm}$, given our interpretation of the different apparent expansions at $6 \mathrm{~cm}$ and $18 \mathrm{~cm}$ for epochs beyond day 1500 . These findings differ from the Bartel et al. (2002) results on the details of the radio structure of SN 1993J and its expansion.

For a distance of $3.63 \mathrm{Mpc}$ to SN 1993J, comparison of our VLBI results with optical spectral line velocities shows that the deceleration is more pronounced in the radio than in the optical. This difference in the deceleration might be due to a progressive penetration of ejecta instabilities into the shocked circumstellar medium, as also suggested by Bartel et al. (2007).

Acknowledgements. The National Radio Astronomy Observatory is a facility of the National Science Foundation operated under cooperative agreement by Associated Universities, Inc. The European VLBI Network is a joint facility for European, Chinese, South African and other radio astronomy institutes funded by their national research councils. Partial support from Spanish grant AyA 2005-08561-C03-02 is ackwnowledged. I.M.V. is a fellow of the Alexander von Humboldt Foundation. K.W.W. wishes to thank the Office of Naval Research Laboratories for the 6.1 funding supporting this research.

\section{Appendix A: The Common Point Method}

Fitting a source model to the visibilities has some advantages: it is possible to describe the information contained in many visibilities with only a few parameters; and it is applied in Fourier 
space, a natural choice for interferometric measurements, thus avoiding the use of deconvolution algorithms that might introduce artifacts in the source structure. However, model fitting to the visibilities also has disadvantages. For example, the fitted parameters depend on the details of the model used. Also, if a model shape is kept fixed while source structure changes take place, the fitting will introduce changing biases. Additionally, there can also be strong coupling between the fitting parameters, and the $\chi^{2}$ to be minimized may have lots of local minima and/or a pathological behavior near the absolute minimum.

Measuring in the sky plane may sometimes (e.g., when the source is very circularly symmetric) simplify matters: the measurement of the source size is made directly on the source image. We describe a new method for estimating the size of a circularly symmetric source in the sky plane without assuming any a priori source model. The algorithm is easy to use and rather insensitive to changes in the internal structure of the observed source.

\section{A.1. Derivation}

We consider a circularly symmetric brightness distribution, $M$, convolved by a Gaussian, $G$, of width $\sigma$. Let $I(\sigma, x, y)$ be the resulting intensity distribution, where $(x, y)$ are the arclengths of the relative right ascension and declination. Then,

$I(\sigma, x, y)=\int_{-\infty}^{\infty} \int_{-\infty}^{\infty} M\left(x^{\prime}, y^{\prime}\right) G\left(x-x^{\prime}, y-y^{\prime}\right) \mathrm{d} x^{\prime} \mathrm{d} y^{\prime}$

where,

$G(x, y)=\frac{1}{2 \pi \sigma^{2}} \exp \left(-\frac{x^{2}+y^{2}}{2 \sigma^{2}}\right)$.

If we express $I(\sigma, x, y)$ in polar coordinates and perform the azimuthal average we have

$$
\begin{aligned}
A v(\sigma, r)= & \exp \left(-\frac{r^{2}}{2 \sigma^{2}}\right) \int_{0}^{\infty} \frac{M\left(r^{\prime}\right)}{\sigma^{2}} \\
& \times \exp \left(-\frac{r^{\prime 2}}{2 \sigma^{2}}\right) \operatorname{BesselI}_{0}\left(-\frac{r r^{\prime}}{\sigma^{2}}\right) r^{\prime} \mathrm{d} r^{\prime},
\end{aligned}
$$

where $A v(\sigma, r)$ is the azimuthal average and BesselI $\mathrm{I}_{0}$ is the modified Bessel function of the first kind. We can consider Eq. (A.3) as the integral transform of the brightness distribution, $M(r)$, in terms of the following kernel function:

$\operatorname{Kernel}\left(\sigma, r, r^{\prime}\right)=\frac{r^{\prime}}{\sigma^{2}} \exp \left(-\frac{r^{2}+r^{\prime 2}}{2 \sigma^{2}}\right) \operatorname{BesselI}_{0}\left(-\frac{r r^{\prime}}{\sigma^{2}}\right)$.

We can now compute the change in $A v(\sigma, r)$ for a small change in $\sigma$. Performing a first-order Taylor expansion with respect to $\delta \sigma$ (i. e., the change in $\sigma$ ), we obtain

$\delta[A v(\sigma, r)]=\frac{\delta \sigma}{\sigma} \int_{0}^{\infty} M\left(r^{\prime}\right) K\left(\sigma, r, r^{\prime}\right) \mathrm{d} r^{\prime}$

with

$$
\begin{aligned}
K\left(\sigma, r, r^{\prime}\right)= & 2 \frac{\exp \left(-\frac{r^{2}+r^{\prime 2}}{2 \sigma^{2}}\right) r^{\prime} \mathrm{d} r^{\prime}}{\sigma^{2}}\left(\left(\frac{r^{2}+r^{\prime 2}}{2 \sigma^{2}}-1\right)\right. \\
& \left.\times \operatorname{BesselI}_{0}\left(\frac{r r^{\prime}}{\sigma^{2}}\right)-\frac{r r^{\prime}}{\sigma^{2}} \operatorname{BesselI}_{1}\left(\frac{r r^{\prime}}{\sigma^{2}}\right)\right) .
\end{aligned}
$$

Thus, we can see from Eq. (A.5) that if there is a value of $r$ (denoted $r_{\mathrm{c}}$ ), directly related to $M$ and $\sigma$, such that

$\int_{0}^{\infty} M\left(r^{\prime}\right) K\left(\sigma, r_{\mathrm{c}}, r^{\prime}\right)=0$,

then the angular average $A v(\sigma, r)$ will not change its value for small changes in the width of the convolving Gaussian for $r=r_{\mathrm{c}}$. We call Common Point $(s)$ those points of $A v(\sigma, r)$ that correspond to such value(s) of $r_{\mathrm{c}}$. For SN 1993J, the values $r_{\mathrm{c}}$ correspond to the abscissas $X_{\mathrm{ICP}}$ and $X_{\mathrm{OCP}}$ shown in Fig. 5 for observations from day 1889 after the explosion.

For the purpose of discussion only, we assume that the profile given by $M(r)$ has a clearly defined cutoff radius, $R$. Then, the integral in Eq. (A.5) will extend from $r^{\prime}=0$ to $r^{\prime}=R$, and $r_{\mathrm{c}}$, multiplied by a factor $C$ that depends only on $\sigma$ and $M(r)$, will equal $R$

$r_{\mathrm{c}} C(\sigma, M)=R$.

If we expand the brightness distribution, $M(r)$, self-similarly by a factor $P$, then

$R \rightarrow P R \Rightarrow M(r) \rightarrow M\left(\frac{r}{P}\right)$

and we arrive at a new expression for Eq. (A.7):

$\int_{0}^{P R} M\left(\frac{r^{\prime}}{P}\right) K\left(\sigma, \frac{r_{\mathrm{c}}}{P}, \frac{r^{\prime}}{P}\right)=0$.

Given that both $r$ and $r^{\prime}$ are scaled by $\sigma$ wherever they appear in the kernel of Eq. (A.6), the radial positions of the Common Points associated with $M\left(\frac{r}{P}\right)$ and $P \sigma$ will be equal to $P$ times the radial positions of the Common Points associated with $M(r)$ and $\sigma$. In other words, given a self-similar expansion of a brightness distribution, the Common Points will expand at the same rate as the brightness distribution, provided that the Gaussians used in the convolutions are also scaled with the source size. In such cases, the relationship between $r_{\mathrm{c}}$ and $R$ would be given by Eq. (A.8), where $C$ would only depend, for the whole expansion, on the profile of the brightness distribution, $M(r)$ and the (constant) ratio between the source radius and the convolving beam. We computed, using $\sigma=0.5 R$, the values of $C$ for a set of possible source distributions, always finding values of $C$ near unity (see Table 2 for six examples of $C$, which we call bias and label as $\left.R / X_{\mathrm{OCP}}\right)$.

Thus, the only condition that must be satisfied for using the Common Point to determine the expansion of SN 1993J is that, for each epoch, the convolving Gaussian beam must be equal to the supernova size multiplied by a given factor, which must be the same for all epochs. The Gaussian beam for each epoch can be found in an iterative way, given that the Common Points are very stable to changes in the convolving beam. We provide the details of this iterative process in the next appendix section.

\section{A.2. Application}

We convolve a map corresponding to a shell-like source of unknown outer radius $R$ with a Gaussian of width $\sigma$. Let us also convolve the same map with Gaussians of different widths given by $\sigma_{i}=H_{i} \sigma$, where the constants $H_{i}$ are all near unity (we use $0.8,0.9,1.1$, and 1.2 ).

If we now compute the azimuthal average of each of these maps and superimpose the results, we find that the profiles cross 
in narrow regions of radial values ${ }^{3}$. The mean value of the radial positions of the crossing points will be an estimate of the radial position of the Common Point described above. Let this estimate be $r_{\mathrm{c}, 1}$.

Now convolve the initial map with a new set of Gaussians, starting with a new $\sigma=F r_{\mathrm{c}, 1}$, where $F$ is a chosen constant (in practice, we choose $F=0.5$ ) and with $\sigma_{i}=H_{i} \sigma$, where the constants $H_{i}$ are the same as in the previous iteration. We now find a new value for the radial position of the Common Point: $r_{\mathrm{c}, 2}$. We can further iterate this procedure to obtain $r_{\mathrm{c}, 3}, r_{\mathrm{c}, 4}, \ldots$ The procedure will soon converge to a value $r_{\mathrm{c}, \mathrm{f}}$. By construction, this value is the common point associated with the brightness profile of the shell and a convolving beam of width equal to $F$ times the (unknown) source radius. Thus, this iterative process gives the radial position of the Common Point associated with a Gaussian beam that scales always by the same factor $F$ with respect to the source radius, regardless of the size of the source.

The Common Point Method works very well with synthetic data. Because of the practical insensitivity of the computed Common Point to the sizes of the Gaussian widths (within reasonable limits, of course), virtually independent of the radial profile of the source structure, the restriction of using $H_{i}$ close to unity can be lifted. Azimuthal averages of VLBI maps of SN 1993J, constructed using Gaussian beamwidths ranging from $\sigma$ to $1.5 \sigma$, will, when superimposed, cross at radial positions that differ by a very small amount (typically, $\sim 1 \mu$ as) (see Fig. 3).

This makes the Common Point Method a robust way of measuring the outer radius of a circularly symmetric, and selfsimilarly expanding, source. It converges in only a few iterations, largely independent of the starting value of $\sigma$.

As a further test of the method, we generated synthetic data at several observing frequencies, with different shell sizes, and for different dynamic ranges. In all cases, the CPM estimates were excellent compared to the true values. We also studied the CPM bias for different (non-homogeneous emission) shell structures, for example, a parabolic-shaped shell (zero at extremes and maximum at center), a double mini-shell (shell with emission only in $R_{\text {in }}$ and $R_{\text {out }}$ ), and a shell with a linear radial decay. In all cases, the CPM bias (i.e., the value of $C$ in Eq. (A.8)) is close to 1 , and is also invariant in a self-similar expansion.

For a real map, we determine $r_{\mathrm{c}, \mathrm{f}}$. Since we know from our testing that $C$ is close to unity, we can very reliably assign the value $r_{\mathrm{c}, \mathrm{f}}$ to $R$. If the expansion is exactly self-similar, the bias $C$ remains constant at all epochs. The accuracy of the source size determination for all epochs is thus translated to an enhanced accuracy in the determination of the expansion curve of the source. Even for slight departures from self-similar expansion, the method remains more accurate than model fitting, according to our testing. If the expansion departs from self-similar, the bias $C$ will slightly change, but will change by much less (usually by a factor of 2 , depending on how the evolution of the brightness profile $M(r)$ differs from self-similarity) than in the model fitting. For example, a change in a shell with no ejecta opacity from a fractional shell width of 0.25 to 0.35 (i.e., a change of $40 \%$ in the fractional shell width) translates into a bias change of only $1.5 \%$ for $X_{\mathrm{OCP}}$ (see Table 2). However, the same change in the fractional shell width would translate into a bias change of around 3\% (depending on the source size; the larger

\footnotetext{
3 As can be seen in Fig. 5, there are two crossing regions, one at the inner edge and the other at the outer edge of the profile. For determining source sizes, we are primarily interested in the outer crossing region.
}
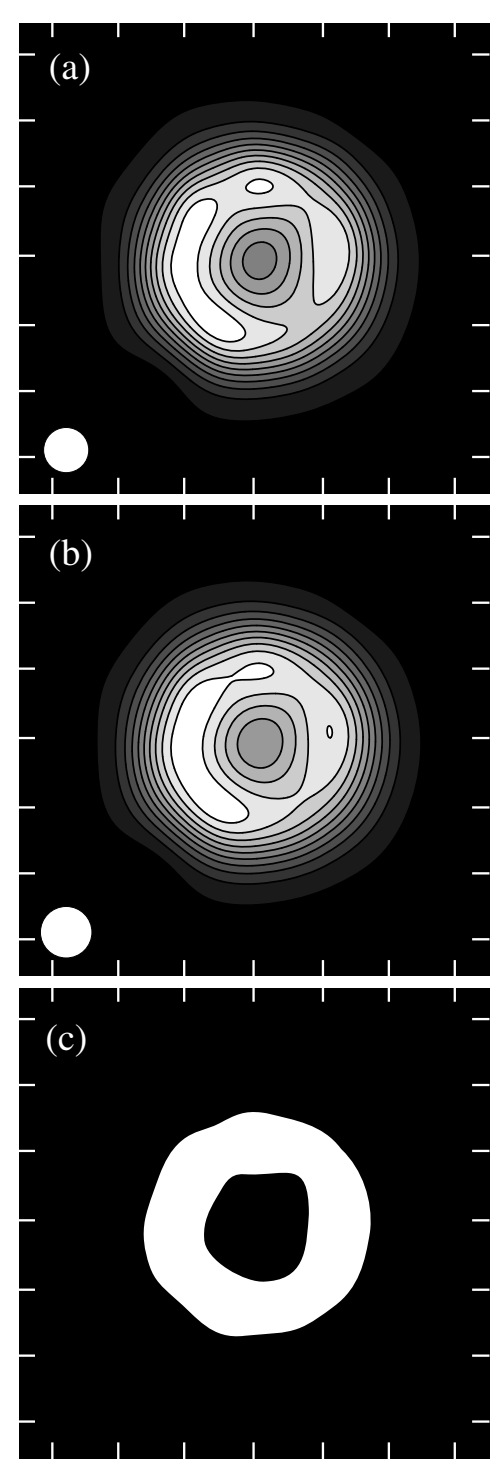

Fig. A.1. $6 \mathrm{~cm}$ contour maps from day 1889 reconstructed using a) a 1.74 mas beam; b) a 1.92 mas beam. The ten contours shown are linearly spaced from 2.56 to $-0.28 \mathrm{mJy}^{-1}$ beam ${ }^{-1}$ for a) and from 2.91 to $-0.32 \mathrm{mJy} \mathrm{beam}^{-1}$ for $\left.\mathbf{b}\right)$. The bottom image, $\mathbf{c}$ ) shows the result of the subtraction of $\mathbf{b}$ ) from a); residuals outside the source have been removed in maps a) and b) prior to subtraction. Image c) was then digitized in flux to 1 bit (positive is white, negative is black). The transition contour of interest in image c) is the outer contour. Tick marks are in units of 2 mas.

the source, the smaller the bias change) if we apply model fitting to the visibilities.

\section{A.3. Source size uncertainties}

The CPM itself does not have a clearly defined way of estimating the source size uncertainty. The remarkable property that all profiles obtained with different beams cross at $X_{\mathrm{OCP}}$ does not have a 2 dimensional equivalent, except for the ideal case of perfect circular symmetry. We assume that the uncertainty in the determination of the source size is related to the departure from circularity of the source. Hence, we assign an uncertainty as described below.

We call "MAP1" the map reconstructed with a beam size of half the radius of SN 1993J (as Fig. A.1a). We then reconstruct 
a map, "MAP2", using a beam size 10\% larger (as Fig. A.1b) than that used to reconstruct MAP1 (the method is almost insensitive to the choice of this percentage). A subtraction of MAP2 from MAP1 will yield "MAP3" (as in Fig. A.1c). To estimate the departure from circularity of the source, we measure from MAP3 the scatter in the radial values of the outer zeroth contour level, $\zeta$, along 800 directions equally distributed in azimuth (the number of directions is arbitrary, and the estimate is insensitive to this choice provided the number is higher than a few hundred). To minimize a possible bias in this measurement between epochs, we reconstruct the map with a pixel size proportional to the beam size, which in turn is proportional to the source size, as mentioned earlier. The standard deviation assigned to the source size measurement is thus $\zeta$.

\section{Appendix B: Visibility modelfitting}

The radial profile of the projection in the sky of the emission from a spherical shell with absorption in its inner side (i.e., from intervening ejecta) is given by the expression

$$
\begin{aligned}
& I(A, R, W, a, r)= \\
& \left\{\begin{aligned}
A\left(\sqrt{R^{2}-r^{2}}-\sqrt{(R W)^{2}-r^{2}}\right)(2-a) / 2 \text { if } \quad r<R W \\
A\left(\sqrt{R^{2}-r^{2}}\right) \text { if } \quad r \geq R W
\end{aligned}\right.
\end{aligned}
$$

where $r$ is the radial coordinate, $R$ is the source radius, $W$ the fractional radius of the inner surface of the shell, $a$ is the degree of absorption ( 0 for no absorption; 1 for total absorption), and $A$ is a scaling factor related to the total shell flux density. The real part of the azimuthal average of the visibilities is fit to $H$, the Hankel transform of $I$, which is equal to the azimuthal average of the Fourier transform of a circularly symmetric source with a profile given by $I$. Thus, the model used in the fitting is

$H(q)=\int_{0}^{\infty} I(A, R, W, a, r) \operatorname{Bessel}_{0}(2 \pi q r) r \mathrm{~d} r$

where $q=\sqrt{u^{2}+v^{2}}$ is the distance in Fourier space. The parameters $A, R, W$, and $a$ are fitted but not necessarily all everytime. We can fix some parameters and fit the others, as explained in several sections of this paper. As said in Sect. 4.4, we apply a radial binning to the visibilities during the azimuthal average and downweight the long baselines with a taper prior to the model fit. We use 300 bins, which cover the first 5 amplitude lobes (see Fig. 1 for a schematic representation of these lobes). For epochs where the supernova was not large enough, the width of the bins is scaled according to the source size, to obtain a similar radial coverage of each averaged visibility (in units of the sizes of the lobes) for all epochs. In Fig. B.1, we show an example of a model fit using $R$ and $A$ as fitting parameters and fixing $a$ to 1 and $W$ to 0.7 (i.e., the fractional shell width to 0.3 ).

\section{Appendix C: Emission structure due to a synchrotron-aged electron population}

The spectrum of the synchrotron emission of an electron with energy $E$ in a magnetic field $B$ is (Pacholczyk 1970)

$I_{v}(B, E) \propto B \cdot \mathrm{F}\left(\sqrt{\frac{3}{2}} \frac{v}{c_{1} B E^{2}}\right)$

where $c_{1}=6.27 \times 10^{18}$ (cgs units) and we assume that $B^{2}=$ $\frac{3}{2} B_{\perp}^{2}, B_{\perp}$ being the magnetic field component orthogonal to the electron velocity.

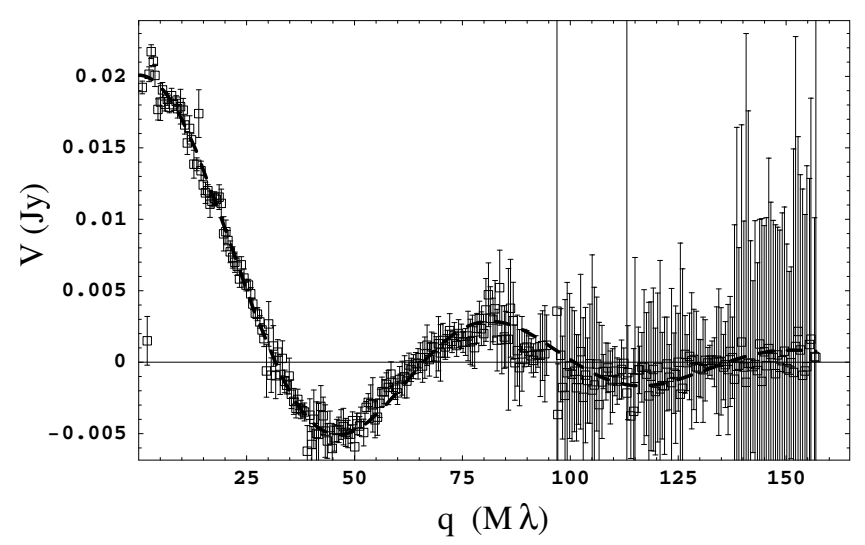

Fig. B.1. Model fit of a spherical shell (of fractional width 0.3 and total absorption, $a=1$ ) to the visibilities, observed on day 1889 after explosion, which have been azimuthally averaged as described in this appendix. The fitting parameters are the source size $R$ and the parameter related to the flux density, $A$. The fitted model is shown as a dashed line. Notice the increase in the error bars for the longest baselines, due to the taper applied.

For large values of $x$, the function $\mathrm{F}(x)$ behaves as follows:

$\mathrm{F}(x) \propto \sqrt{x} \cdot \exp (-x)$.

Thus, for small enough values of $B E^{2}$, the ratio of emission at frequencies $v_{1}>v_{2}$ is

$\frac{I_{v_{1}}(E)}{I_{v_{2}}(E)}=\sqrt{\frac{v_{1}}{v_{2}}} \cdot \exp \left(\sqrt{\frac{3}{2}} \frac{\left(v_{2}-v_{1}\right)}{c_{1} B E^{2}}\right)$.

As shown, this intensity ratio depends on the strength of the magnetic field. If we now consider the spectrum resulting from integrating over all energies using an electron energy distribution of the canonical type $N \propto E^{-p}$, the intensities $I_{v}$ at different frequencies will be such that the dependence of the intensity ratio on the magnetic field will vanish since the resulting spectrum will depend separately on $B$ and $v$. However, this separation would not hold were the distribution lower than the canonical at high energies as in synchrotron aging. In this case, after integration over all electron energies, the intensity ratio depends on the magnetic field. Thus, a radially changing magnetic field would translate into a radially changing ratio of emission intensities at different frequencies.

For day 3200 after explosion, Chandra et al. (2004) inferred a spectral index $\alpha$ that is steeper at higher frequencies than at lower frequencies, i.e.,

$\alpha=\left\{\begin{array}{l}0.51 v \leq 4 \mathrm{GHz} \\ 1.13 v>4 \mathrm{GHz}\end{array}\right.$

We now compute the electron distribution $N(E)$ that reproduces the spectrum measured by Chandra et al. (2004) and obeys Eq. (1). The power index, $p$, of the energy distribution is related to the spectral index, $\alpha$, of the emission spectrum by the expression $p=1+2 \alpha$ (Pacholczyk 1970). We can now integrate over all energies to obtain the contribution of all the electrons to the emission intensity at each distance $D$ (distance from the contact discontinuity) and radiofrequency $v$ :

$S_{v}(D)=\int_{E_{\min }}^{\infty} I_{v}(B(D), E) N(E) \mathrm{d} E$. 
The normalized profile of this emission intensity is shown in Fig. 10 for 6 and $18 \mathrm{~cm}$. For a given value of $D$, the emission at the longer wavelength is always higher than the emission at the shorter wavelength. In other words, for a given intensity level, the emission reaches further out at the longer wavelength.

\section{References}

Alberdi, A., \& Marcaide, J. M. 2005, in Cosmic Explosions, ed. J. M. Marcaide \& K. W. Weiler (Berlin: Springer), IAU Colloq., 192, 23

Aldering, G., Humphreys, R. M., \& Richmond, M. 1994, AJ, 107, 662

Barbon, R., Benetti, S., Cappellaro, E., et al. 1995, A\&A, 110, 513

Baron, E., Hauschildt, P. H., Branch, D., et al. 1993, ApJ, 416, 21

Baron, E., Hauschildt, P. H., \& Branch, D. 1994, ApJ, 426, 334

Baron, E., Hauschildt, P. H., Branch, D., et al. 1995, ApJ, 441, 170

Bartel, N., Bietenholz, M. F., Rupen, M. P., et al. 1994, Nature, 368, 610

Bartel, N., Bietenholz, M. F., Rupen, M. P., et al. 2000, Science, 287, 112

Bartel, N., Bietenholz, M. F., Rupen, M. P., et al. 2002, ApJ, 581, 404

Bartel, N., Bietenholz, M. F., Rupen, M. P., \& Dwarkadas, V. V. 2007, ApJ, 668, 924

Bartunov, O. S., Blinnikov, S. I., Pavlyuk, N. N., \& Tsvetkov, D. Yu 1994, A\&A, 281,53

Bietenholz, M. F., Bartel, N., \& Rupen, M. P. 2001, ApJ, 557, 770

Bietenholz, M. F., Bartel, N., \& Rupen, M. P. 2003, ApJ, 597, 374

Bietenholz, M. F., Bartel, N., \& Rupen, M. P. 2005, in Cosmic Explosions, ed

J. M. Marcaide, \& K. W. Weiler (Berlin: Springer), IAU Colloq., 192, 23

Blondin, J. M., Lundqvist, P., \& Chevalier, R. A. 1996, ApJ, 472, 257

Branch, D., Jeffery, D. J., Blaylock, M., \& Hatano, K. 2000, PASP, 112, 217

Chandra, P., Ray, A., \& Bhatnagar, S. 2004, 604, 97

Chevalier, R. A. 1982a, ApJ, 258, 790

Chevalier, R. A. 1982b, ApJ, 259, 302

Chevalier, R. A., \& Blondin, J. M. 1995, ApJ, 444, 312

Clocchiatti, A., Wheeler, J. C., Barker, E. S., et al. 1995, ApJ, 446, 167

Filippenko, A. V. 1988, AJ, 96, 1941

Filippenko, A. V., Matheson, T., \& Ho, L. C. 1993, ApJ, 415, 103

Filippenko, A. V., Matheson, T., \& Barth, A. J. 1994, AJ, 108, 2220

Finn, R. A., Fesen, R. A., Darling, G. W., Thorstensen, J. R., \& Worthey, G. S. 1995, AJ, 110, 300

Fransson, C. 1984, A\&A, 132, 115

Fransson, C., \& Björnsson, C.-I. 1998, ApJ, 509, 861

Fransson, C., Lundqvist, P., \& Chevalier, R. A. 1996, ApJ, 461, 993

Fransson, C., Challis, P. M., Chevalier, R. A., et al. 2005, ApJ, 622, 991

Freedman, W. L., Hughes, S.M., Madore, B.F., et al. 1994, ApJ, 427, 628

Garnavich, P. M., \& Ann, H. B. 1994, AJ, 108, 1002

Gill., E., \& Murray, W. 1978, SIAM J. Numer. Anal., 15, 977

Hashimoto, M., Iwamoto, K., \& Nomoto, K. 1993, ApJ, 414, 105

Höflich, P., Langer, N., \& Dushinger, M. 1993, A\&A, 275, 29

Houck, J. C., \& Fransson, C. 1996, ApJ, 456, 811

Jeffery, D. J., Krishner, R. P., Challis, P. M., et al. 1994, ApJ, 421, 27

Kohmura, Y., Inoue, H., Aoki, T., et al. 1994, PASJ, 46, 157

Lewis, J. R., Walton, N. A., Meikle, W. P. S., et al. 1994, MNRAS, 266, 27

Li, A., Hu, J., Wang, L., Jiang, X., \& Li, H. 1994, Ap\&SS, 211, 323
Marcaide, J. M. 2005, in 1604-2004: Supernovae as Cosmological Lighthouses, ed. M. Turatto, S. Benetti, L. Zampieri, \& W. Shea (San Francisco: ASP Conf. Ser.), 298

Marcaide, J. M., Alberdi, A., Elósegui, P., et al. 1994, ApJ, 424, 25

Marcaide, J. M., Alberdi, A., Ros, E., et al. 1995a, Nature, 373, 44

Marcaide, J. M., Alberdi, A., Ros, E., et al. 1995b, Science, 270, 1475

Marcaide, J. M., Alberdi, A., Ros, E., et al. 1997, ApJ, 486, 31

Marcaide, J. M., Martí-Vidal, I., Ros, E., et al. 2005, in Cosmic Explosions, ed. J. M. Marcaide, \& K. W. Weiler (Berlin: Springer), IAU Colloq., 192, 29

Martí-Vidal, I. 2008, Ph.D. Thesis, University of Valencia, Spain

Matheson, T., Filippenko, A. V., Ho, L. C., Barth, A. J., \& Leonard, D. C., 2000a, AJ, 120, 1499

Matheson, T., Filippenko, A. V., Ho, L. C., Barth, A. J., \& Leonard, D. C. 2000b, AJ, 120, 1487

Maund, J. R., Smartt, S. J., Kudritzki, R. P., Podsiadlowski, P., \& Gilmore, G. F. 2004, Nature, 427, 129

Mioduszewski, A. J., Dwarkadas, V. V., \& Ball, L. 2001, ApJ, 562, 869

Nomoto, K., Suzuki, T., Shigeyama, T., et al. 1993, Nature, 364, 507

Pacholczyk, A. N. 1970, Radio Astrophys., 1st edn. (San Francisco: Freeman \& Co)

Patat, F., Chugai, N., \& Mazzali, P. A. 1995, A\&A, 299, 715

Pérez-Torres, M. A., Alberdi, A., \& Marcaide, J. M. 2001, A\&A, 374, 997

Pérez-Torres, M. A., Alberdi, A., \& Marcaide, J. M. 2002, A\&A, 394, 71

Podsiadlowski, P., Hsu, J. J. L., Joss, P. C., \& Ross, R. R. 1993, Nature, 364, 509

Pooley, G. G., \& Green, D. A. 1993, MNRAS, 264, 17

Ray, A., Singh, K. P., \& Sutaria, F. K. 1993, JApA, 14, 53

Ripero, J., \& García, F. 1993, IAU Circ., 5731

Rupen, M. P., Beasley, A. J., Bartel, N., et al. 1998, in Radio Emission from Galactic and Extragalactic Compact Sources, ed. J. A. Zensus, G. B. Taylor, \& J. M. Wrobel, IAU Colloq., 164

Schmidt, B. P., Kirshner, R. P., Eastman, R. G., et al. 1993, Nature, 364, 600

Shepherd, M. C., Pearson, T. J., \& Taylor, G. B. 1995, BAAS, 26, 987

Shigeyama, T., Suzuki, T., Kumagai, S., et al. 1994, ApJ, 420, 341

Spyromilio, J. 1994, MNRAS, 266, 61

Suzuki, T., \& Nomoto, K. 1995, ApJ, 455, 658

Swartz, D. A., Clocchiatti, A., Benjamin, R., Lester, D. F., \& Wheeler, J. C. 1993, Nature, 365, 232

Taniguchi, Y., Murayama, T., Sato, et al. 1993, PASJ, 45, 43

Trammell, S. R., Hines, D. C., \& Wheeler, J. C. 1993, ApJ, 414, 21

Tran, H. D., Filippenko, A. V., Schmidt, G. D., et al. 1997, PASP, 109, 489

Utrobin, V. 1994, A\&A, 281, 89

Utrobin, V. 1996, A\&A, 306, 219

Van Dyk, S. D., Weiler, K. W., Sramek, R. A., Rupen, M. P., \& Panagia, N. 1994, ApJ, 432, L115

Wang, L., \& Hu, J. 1994, Nature, 369, 380

Weiler, K. W., Williams, C. L., Panagia, N., et al. 2007, ApJ, 671, 1959

Wheeler, J. C., Barker, E., Benjamin, R., et al. 1993, ApJ, 417, 71

Wolfram, S. 2003, The Mathematica book, 5th edn. (Wolfram Media)

Woosley, S. E., Pinto, P. A., Martin, P. G., \& Weaver, T. A. 1987, ApJ, 318, 664

Woosley, S. E., Eastman, R. G., Weaver, T. A., \& Pinto, P. A. 1994, ApJ, 429, 300

Young, T. R., Baron, E., \& Branch, D., 1995, ApJ, 449, 51

Zimmerman, H. U., Lewin, W., Predehl, P., et al. 1994, Nature, 367, 621 